TRANSACTIONS OF THE

AMERICAN MATHEMATICAL SOCIETY

Volume 349, Number 3, March 1997, Pages 903-934

S 0002-9947(97)01904-1

\title{
A CHARACTERIZATION OF FINITELY DECIDABLE CONGRUENCE MODULAR VARIETIES
}

\author{
PAWEE M. IDZIAK
}

\begin{abstract}
For every finitely generated, congruence modular variety $\mathcal{V}$ of finite type we find a finite family $\mathcal{R}$ of finite rings such that the variety $\mathcal{V}$ is finitely decidable if and only if $\mathcal{V}$ is congruence permutable and residually small, all solvable congruences in finite algebras from $\mathcal{V}$ are Abelian, each congruence above the centralizer of the monolith of a subdirectly irreducible algebra $\mathbf{A}$ from $\mathcal{V}$ is comparable with all congruences of $\mathbf{A}$, each homomorphic image of a subdirectly irreducible algebra with a non-Abelian monolith has a non-Abelian monolith, and, for each ring $R$ from $\mathcal{R}$, the variety of $R$-modules is finitely decidable.
\end{abstract}

The aim of this paper is to prove the following characterization of finitely decidable finitely generated congruence modular varieties of finite type.

Main Theorem. Let $\mathcal{V}$ be a finitely generated congruence modular variety of finite type. Then $\mathcal{V}$ is finitely decidable if and only if the following conditions hold:

$(\mathbf{m} .1) \mathcal{V}$ is congruence permutable,

$(\mathbf{m . 2}) \mathcal{V}$ is residually small,

(m.3) if $\mathbf{A}$ is a subdirectly irreducible algebra in $\mathcal{V}$ and $\nu$ is the centralizer of the monolith of $\mathbf{A}$, then

(m.3.1) $\nu$ is the largest solvable congruence of $\mathbf{A}$,

(m.3.2) $\nu$ is an Abelian congruence,

(m.3.3) $\nu$ is comparable with all congruences of $\mathbf{A}$,

(m.3.4) the congruences of $\mathbf{A}$ above $\nu$ are linearly ordered,

(m.3.5) $\mathbf{A} / \nu$ has no homomorphic image with Abelian monolith,

$(\mathbf{m} .4)$ for each finite algebra $\mathbf{D}$ from $\mathcal{V}$ having linearly ordered congruences and non-Abelian monolith, the variety $\mathcal{V}_{\mathbf{D}}$ of $R(\mathcal{V} * \mathbf{D},|D|)$-modules (expanded by some constants) is finitely decidable, where $\mathcal{V} * \mathbf{D}$ denotes the subvariety of $\mathcal{V}$ generated by all subdirectly irreducible algebras $\mathbf{A}$ of $\mathcal{V}$ such that $\mathbf{A} / \nu$ is isomorphic to $\mathbf{D}$.

The rings $R(\mathcal{V} * \mathbf{D},|D|)$ as well as the varieties $\mathcal{V}_{\mathbf{D}}$ are defined in Section 2 . Our condition (m.4) although slightly complicated can be actually understood as a reduction of finite decidability of a given variety $\mathcal{V}$ to the finite decidability of the finite family of varieties of modules determined by $\mathcal{V}$. Moreover this reduction

Received by the editors January 26, 1993 and, in revised form, January 15, 1994.

1991 Mathematics Subject Classification. Primary 03B25, 08A05; Secondary 03C13, 08B10, $08 \mathrm{~B} 26$.

Key words and phrases. Finite decidability, structure theory, congruence modularity.

Research partially supported by KBN Grant No. 2 P301-029-04 and Fulbright Grant No. 17381.

(C)1997 American Mathematical Society 
is effective. Given a finitely generated congruence modular variety $\mathcal{V}$ (of finite type), say $\mathcal{V}=H S P(\mathbf{A})$ for some finite $\mathbf{A}$, we can check whether $\mathcal{V}$ is congruence permutable by inspecting all ternary terms from the (finite) 3-freely generated algebra in $\mathcal{V}$. Next, to ensure that $\mathcal{V}$ is residually small it suffices (see Remark 8.9 of [7]) to check whether each subalgebra of $\mathbf{A}$ satisfies the congruence identity $x \wedge[y, y]=[x \wedge y, y]$. If $\mathcal{V}$ is residually small then all its subdirectly irreducible algebras are not bigger than $|A|+\left(|A|^{|A|^{|A|+3}}\right)$ ! (see Theorem 10.15 of [7]). Thus after listing all of them we can verify all conditions $(\mathbf{m . 3 . 1})-(\mathbf{m . 3 . 5})$. If we are successful up to now, we produce (as described in Section 2) finite rings $R(\mathcal{V} * \mathbf{D},|D|)$ and finite sets of finite modules generating the varieties $\mathcal{V}_{\mathbf{D}}$. Now $\mathcal{V}$ is finitely decidable if and only if all of the $\mathcal{V}_{\mathbf{D}}$ 's are finitely decidable.

\section{INTRODUCTION}

The study of the decision problem for various classes of structures began in the 1930's when Church gave the first undecidability result.

In Universal Algebra one primarily studies collections of algebras known as varieties (that is classes of algebras closed under subalgebras, products and homomorphic images). Over the past few decades there has been a systematic attempt to classify decidable varieties.

Soon after the appearance of the undecidability result of Church and Rosser, namely that Peano's arithmetic is undecidable, A.Tarski took over and developed in 1938-39 a general method for proving the undecidability of first-order theories. The method was used by him and many others to obtain a lot of examples of undecidable varieties including (non-Abelian) groups, (commutative) semigroups, (distributive) lattices, monadic algebras and varieties of algebras with two unary operations.

On the other hand A.Tarski [24] proved (in 1949) the decidability of the class of Boolean algebras. Another important result of this kind is the one of W.Szmielew ([23], 1955), saying that any variety of Abelian groups is decidable. These, together with the decidability of algebras with one unary operation (the decidability of which was shown by A.Ehrenfeucht [6], 1959) form, as it turned out recently, the paradigms for decidable varieties.

All of these results answer the question of whether a particular variety is decidable. However there were no general theorems concerning the structure of decidable varieties. The situation changed in the middle of the 1970's when H.Werner [26] extended a sheaf technique, used earlier by S.D.Comer [5] for monadic algebras, to prove that each finitely generated discriminator variety has a decidable first order theory. Simultaneously A.P.Zamyatin $[29,30]$ gave a complete characterization of decidable varieties of groups and rings. Excellent results of S.D.Comer, H.Werner and A.P.Zamyatin were used by S.Burris and R.McKenzie ([2], 1981) to describe the structure of decidable locally finite congruence-modular varieties.

Recently R.McKenzie and M.Valeriote ([19], 1989) were able to drop the assumption of congruence modularity, describing the structure of decidable varieties in the locally finite setting. They reduced the problem of determining the decidable locally finite varieties to two special questions: which varieties of modules over finite rings are decidable, and which discriminator varieties are decidable.

A discriminator variety is a variety of algebras that can be thought of as Boolean algebras with extra operators. Using sophisticated sheaf constructions S.Burris, 
R.McKenzie, M.Valeriote [3] along with R.Willard [25, 27], have recently made significant progress in the study of decidable discriminator varieties.

Decidable varieties are really quite rare. They, if locally finite, were completely characterized in terms of the behavior of congruences of their algebras. Birkhoff's suggestion in the 1930's that congruence lattices should be considered as fundamental associated structures has proved to be remarkably farsighted. In particular, the mentioned results of S.Burris, R.McKenzie and M.Valeriote justified an old standing supposition that decidability of a variety and nice structure theory go hand in hand. In case a variety is finitely generated and decidable then all its algebras can be recovered, up to isomorphism, from their congruence lattices and a single finite algebra.

The above connections between the structure of algebras and behavior of their congruences is extremely strong if one restricts to the class of finite algebras. This leads to the question of finite decidability of a given class of algebras - a variety is said to be finitely decidable if the theory of its finite members is recursive. Although many undecidable varieties are also finitely undecidable, these two notions are completely independent - any of the four possible cases can occur. Boolean algebras are both decidable and finitely decidable while distributive lattices are undecidable and finitely undecidable. From the work of A.P.Zamyatin [30] it follows that the variety of rings generated by the ring of integers modulo 4 is undecidable but finitely decidable. The most interesting example was found by J.Jeong [14] who constructed a decidable discriminator variety that is finitely undecidable.

For a better understanding of this situation one needs to have a deeper look into the structure of finitely decidable varieties. In a series of papers $[10,11,12]$ a complete description of those finitely decidable varieties that are finitely generated and congruence distributive is given. Such a variety is finitely decidable if and only if it is congruence permutable and the congruence lattice of each (finite) subdirectly irreducible algebra in the variety is linearly ordered. Both these conditions were proved to be necessary in a locally finite setting.

Very recently J.Jeong [14] (see also $[15,16]$ ) made a profound study of the finitely decidable varieties which are locally finite and congruence modular. He found several properties such a variety has to enjoy. The list of necessary conditions was extended by M.Valeriote and the author in [13].

In this paper the list is further extended, and the obtained extension is shown to be complete.

The reader is assumed to be well grounded in modern logic and universal algebra. For all necessary concepts on the decision problems we refer to the survey article [22]. A nice exposition of the method of semantical interpretation can be found in [2]. Our universal algebraic terminology and notation will generally follow those of [4] and [7].

\section{Modules Associated with Algebras}

The main tool used in the proofs of our results is the commutator theory. Thus we assume the reader's familiarity with [7]. In their study of congruence modular varieties Ralph Freese and Ralph McKenzie [7] defined a family of rings $R(\mathcal{V}, \lambda)$, where $\lambda$ is a nonzero cardinal number, such that a lot of information about the algebras from the variety $\mathcal{V}$ can be inferred from the behavior of the $R(\mathcal{V}, \lambda)$ modules. In this section we briefly recall the definition of the rings $R(\mathcal{V}, \lambda)$ and 
properties of $R(\mathcal{V}, \lambda)$-modules which we will need in our considerations. For our purposes it suffices to restate these results only for finite $\lambda$.

Fix a congruence modular variety $\mathcal{V}$ with a Gumm term $\mathbf{p}(x, y, z)$. Let $\mathbf{A}$ be any algebra from $\mathcal{V}$ and let $\beta$ be an Abelian congruence of $\mathbf{A}$. Then for any element $d \in A$ the equivalence class $d / \beta$ can be endowed with operations:

$$
\begin{aligned}
a+b & =\mathbf{p}(a, d, b), \\
-a & =\mathbf{p}(d, a, d),
\end{aligned}
$$

such that $M_{\mathbf{A}}(\beta, d)=(d / \beta,+,-, d)$ is a commutative group.

Now let $n$ be any positive integer and let $\mathbf{F}$ be the free algebra in $\mathcal{V}$ freely generated by the set $X=\left\{u, v_{0}, \ldots, v_{n-1}\right\}$. Denote by $\theta_{i}$ the principal congruence of $\mathbf{F}$ generated by the pair $\left(u, v_{i}\right)$. Since the congruence $\theta_{i}$ is Abelian over $\left[\theta_{i}, \theta_{i}\right]$, $\bar{H}_{i j}=\left\{r /\left[\theta_{i}, \theta_{i}\right]: r \in v_{j} / \theta_{i}\right\}$ is an Abelian group where $v_{j} /\left[\theta_{i}, \theta_{i}\right]$ is the zero element and the operations,+- are defined by

$$
\begin{aligned}
\left(r /\left[\theta_{i}, \theta_{i}\right]\right)+\left(s /\left[\theta_{i}, \theta_{i}\right]\right) & =\mathbf{p}\left(r, v_{j}, s\right) /\left[\theta_{i}, \theta_{i}\right], \\
-\left(r /\left[\theta_{i}, \theta_{i}\right]\right) & =\mathbf{p}\left(v_{j}, r, v_{j}\right) /\left[\theta_{i}, \theta_{i}\right],
\end{aligned}
$$

for any $r=r(u, \mathbf{v}), s=s(u, \mathbf{v}) \in v_{j} / \theta_{i}$.

Moreover the set $R(\mathcal{V}, n)$ of all $(n \times n)$-matrices $\left(m_{i j}\right)$ with $m_{i j} \in \bar{H}_{j i}$ treated as a product of the family $\left\{\bar{H}_{i j}: i, j<n\right\}$ of Abelian groups is an Abelian group where zero is the matrix whose $(i, j)$-th element is $v_{i} /\left[\theta_{j}, \theta_{j}\right]$.

The second step R.Freese and R.McKenzie have done here lies in introducing the multiplication of matrices from $R(\mathcal{V}, n)$ in such a way that $R(\mathcal{V}, n)$ becomes a ring with unit. This multiplication is the ordinary matrix multiplication where the product of $t /\left[\theta_{j}, \theta_{j}\right]$ and $r /\left[\theta_{i}, \theta_{i}\right]$ for any $t=t(u, \mathbf{v}) \in v_{k} / \theta_{j}$ and $r=r(u, \mathbf{v}) \in v_{j} / \theta_{i}$ is given by

$$
\left(t /\left[\theta_{j}, \theta_{j}\right]\right) \cdot\left(r /\left[\theta_{i}, \theta_{i}\right]\right)=t(r, \mathbf{v}) /\left[\theta_{i}, \theta_{i}\right] .
$$

The reader is referred to Chapter IX of [7] for details.

In our further considerations we will also need the matrices $e_{l}$, where $l<n$, whose $(i, j)$-th element is $u /\left[\theta_{l}, \theta_{l}\right]$ for $i=j=l$ and $v_{i} /\left[\theta_{j}, \theta_{j}\right]$ otherwise. It should be clear that the $e_{l}$ 's are pairwise orthogonal idempotents that sum to the unit of the ring $R(\mathcal{V}, n)$.

Now let $\mathbf{A}$ be a finite algebra in $\mathcal{V}, \beta$ be an Abelian congruence of $\mathbf{A}$ and $A / \beta=\left\{d_{0} / \beta, \ldots, d_{n-1} / \beta\right\}$. The last step is to determine the action of the ring $R(\mathcal{V}, n)$ on the direct sum $M_{\mathbf{A}}(\beta)$ of the Abelian groups $M_{\mathbf{A}}\left(\beta, d_{i}\right), \quad i<n$. Take $\mathbf{a}=\left(a_{0}, \ldots, a_{n-1}\right) \in M_{\mathbf{A}}(\beta)$ and $\mathbf{m}=\left(r_{i j} /\left[\theta_{j}, \theta_{j}\right]\right) \in R(\mathcal{V}, n)$ with $r_{i j}=$ $r_{i j}\left(u, v_{0}, \ldots, v_{n-1}\right) \in v_{i} / \theta_{j}$. The $i$-th element of $\mathbf{m} \cdot \mathbf{a}$ is

$$
\sum_{j<n} r_{i j}\left(a_{j}, d_{0}, \ldots, d_{n-1}\right) \text {. }
$$

Once more we refer to [7] for details of this action under which $M_{\mathbf{A}}(\beta)$ is a unitary $R(\mathcal{V}, n)$-module.

As was said at the very beginning, this action carries a lot of information about the algebra $\mathbf{A}$ itself.

First of all note that for any $i, j=0, \ldots, n-1$ and any group homomorphism

$$
h: M_{\mathbf{A}}\left(\beta, d_{i}\right) \longrightarrow M_{\mathbf{A}}\left(\beta, d_{j}\right)
$$

given by the restriction of a unary polynomial of $\mathbf{A}$, there is an element $r=r(u, \mathbf{v}) \in$ $v_{j} / \theta_{i}$ such that $h(a)=r\left(a, d_{0}, \ldots, d_{n-1}\right)$ for all $a \in M_{\mathbf{A}}\left(\beta, d_{i}\right)$. 
Now observe that for any fundamental operation $\mathbf{f}$, say $k$-ary, and all $a_{1}, \ldots, a_{k}$ $\in A$ with $a_{j} \in M_{\mathbf{A}}\left(\beta, d_{i_{j}}\right)$ we have

$$
\begin{aligned}
\mathbf{f}\left(a_{1}, \ldots, a_{k}\right) & =\mathbf{f}\left(d_{i_{1}}, \ldots, d_{i_{k}}\right) \\
& +\sum_{j=1}^{k}\left(\mathbf{f}\left(d_{i_{1}}, \ldots, d_{i_{j-1}}, a_{j}, d_{i_{j+1}}, \ldots, d_{i_{k}}\right)-\mathbf{f}\left(d_{i_{1}}, \ldots, d_{i_{k}}\right)\right) .
\end{aligned}
$$

Now, for $d_{i_{0}} \in\left\{d_{0}, \ldots, d_{n-1}\right\}$ chosen such that $\mathbf{f}\left(d_{i_{1}}, \ldots, d_{i_{k}}\right) \in M_{\mathbf{A}}\left(\beta, d_{i_{0}}\right)$, the mapping $M_{\mathbf{A}}\left(\beta, d_{i_{j}}\right) \longrightarrow M_{\mathbf{A}}\left(\beta, d_{i_{0}}\right)$ that associates with each $x$ from $M_{\mathbf{A}}\left(\beta, d_{i_{j}}\right)$ the element

$$
\mathbf{f}\left(d_{i_{1}}, \ldots, d_{i_{j-1}}, x, d_{i_{j+1}}, \ldots, d_{i_{k}}\right)-\mathbf{f}\left(d_{i_{1}}, \ldots, d_{i_{k}}\right)
$$

of $M_{\mathbf{A}}\left(\beta, d_{i_{0}}\right)$, is a group homomorphism given by a unary polynomial. Identifying each element $a \in d_{i} / \beta$ with the tuple $\left(d_{0}, \ldots, d_{i-1}, a, d_{i+1}, \ldots, d_{n-1}\right)$ from the $R(\mathcal{V}, n)$-module $M_{\mathbf{A}}(\beta)$ and using the previous remark we know that there are mappings

$$
\mathbf{f}^{(M)}:\left\{d_{0} / \beta, \ldots, d_{n-1} / \beta\right\}^{k} \longrightarrow M_{\mathbf{A}}(\beta)
$$

and for each $j=1, \ldots, k$

$$
\mathbf{f}^{(j)}:\left\{d_{0} / \beta, \ldots, d_{n-1} / \beta\right\}^{k} \longrightarrow R(\mathcal{V}, n)
$$

such that

$$
\mathbf{f}\left(a_{1}, \ldots, a_{k}\right)=\left(\sum_{j=1}^{k} \mathbf{f}^{(j)}\left(d_{i_{1}} / \beta, \ldots, d_{i_{k}} / \beta\right) \cdot a_{j}\right)+\mathbf{f}^{(M)}\left(d_{i_{1}} / \beta, \ldots, d_{i_{k}} / \beta\right),
$$

whenever $a_{1} \stackrel{\underline{\beta}}{=} d_{i_{1}}, \ldots, a_{k} \stackrel{\underline{\beta}}{=} d_{i_{k}}$.

Note here that if $\mathbf{f}\left(d_{i_{1}}, \ldots, d_{i_{k}}\right) \in M_{\mathbf{A}}\left(\beta, d_{j}\right)$ then $\mathbf{f}^{(M)}\left(d_{i_{1}} / \beta, \ldots, d_{i_{k}} / \beta\right)$ is a vector $\left(u_{0}, \ldots, u_{n-1}\right)$ in the module $M_{\mathbf{A}}(\beta)=M_{\mathbf{A}}\left(\beta, d_{0}\right) \times \ldots \times M_{\mathbf{A}}\left(\beta, d_{n-1}\right)$ for which $u_{j}=\mathbf{f}\left(d_{i_{1}}, \ldots, d_{i_{k}}\right)$ and $u_{i}=d_{i}$ for $i \neq j$.

We are going to recover the algebra $\mathbf{A}$ from its quotient $\mathbf{D}=\mathbf{A} / \beta$ and the $R(\mathcal{V},|D|)$-module $M_{\mathbf{A}}(\beta)$. Obviously the constants

$$
\mathbf{f}^{(j)}\left(d_{i_{1}} / \beta, \ldots, d_{i_{k}} / \beta\right) \in R(\mathcal{V},|D|)
$$

as well as $\mathbf{f}^{(M)}\left(d_{i_{1}} / \beta, \ldots, d_{i_{k}} / \beta\right) \in M_{\mathbf{A}}(\beta)$ are of a big help since they allow us to recover the fundamental operations of the algebra $\mathbf{A}$. However, first we need to recover the universe of $\mathbf{A}$. To do this, note that the universe of $\mathbf{A}$ can be identified with the following subset of $D \times M_{\mathbf{A}}(\beta)$

$$
\left\{\left(d_{l} / \beta, \mathbf{m}\right) \in D \times M_{\mathbf{A}}(\beta): \mathbf{m}_{j}=0 \text { for all } j \neq l\right\} .
$$

A careful inspection of the action of the ring $R(\mathcal{V},|D|)$ on $M_{\mathbf{A}}(\beta)$ allows us to claim that for the matrix $e_{l} \in R(\mathcal{V},|D|)$ previously defined, we have for all $\mathbf{m} \in M_{\mathbf{A}}(\beta)$

$$
e_{l} \cdot \mathbf{m}=\mathbf{m} \text { iff } \mathbf{m}_{j}=0 \text { for all } j \neq l .
$$

This leads to the following definitions.

Definition 1. Let $\mathbf{D} \in \mathcal{V}$ be a finite algebra with the universe $\left\{d_{0}, \ldots, d_{n-1}\right\}$. Define

$$
\mathbf{e}: D \longrightarrow R(\mathcal{V}, n)
$$

by putting $\mathbf{e}\left[d_{i}\right]=e_{i}$. 
Now, for every $k$-ary fundamental operation $\mathbf{f}$ of $\mathcal{V}$ and $j=1, \ldots, k$ we define a mapping

$$
\mathbf{f}^{(j)}: D^{k} \longrightarrow R(\mathcal{V}, n) \text {. }
$$

For $\left(d_{i_{1}}, \ldots, d_{i_{k}}\right) \in D^{k}$ we need to define an element $\mathbf{f}^{(j)}\left[d_{i_{1}}, \ldots, d_{i_{k}}\right]$ of the ring $R(\mathcal{V}, n)$, i.e. an $n \times n$ matrix

$$
\left(\mathbf{f}_{p, q}^{(j)}\left[d_{i_{1}}, \ldots, d_{i_{k}}\right] /\left[\theta_{q}, \theta_{q}\right]\right)_{p, q<n},
$$

where

$$
\mathbf{f}_{p, q}^{(j)}\left[d_{i_{1}}, \ldots, d_{i_{k}}\right]=\mathbf{f}_{p, q}^{(j)}\left[d_{i_{1}}, \ldots, d_{i_{k}}\right]\left(u, v_{0}, \ldots, v_{n-1}\right)
$$

is a term from $v_{p} / \theta_{q}$, i.e. $\mathcal{V}$ satisfies the identity

$$
\mathbf{f}_{p, q}^{(j)}\left[d_{i_{1}}, \ldots, d_{i_{k}}\right]\left(v_{q}, v_{0}, \ldots, v_{n-1}\right)=v_{p}
$$

Put

$$
\begin{aligned}
& \mathbf{f}_{p, q}^{(j)}\left[d_{i_{1}}, \ldots, d_{i_{k}}\right]\left(u, v_{0}, \ldots, v_{n-1}\right) \\
& \quad= \begin{cases}\mathbf{p}\left(\mathbf{f}\left(v_{i_{1}}, \ldots, u, \ldots, v_{i_{k}}\right), \mathbf{f}\left(v_{i_{1}}, \ldots, v_{i_{k}}\right), v_{p}\right), & \text { if } q=i_{j} \\
& \text { and } \mathbf{f}\left(d_{i_{1}}, \ldots, d_{i_{k}}\right)=d_{p}, \\
v_{p}, & \text { otherwise, }\end{cases}
\end{aligned}
$$

with $u$ occurring at the $j$-th place.

From this definition we know that the matrix $\mathbf{f}^{(j)}\left[d_{i_{1}}, \ldots, d_{i_{k}}\right]$ has at most one non-zero entry which can occur at the position with coordinates (number of $\mathbf{f}\left(d_{i_{1}}, \ldots, d_{i_{k}}\right)$, number of $\left.d_{i_{j}}\right)$. This gives that

$$
\mathbf{e}\left[\mathbf{f}\left(d_{i_{1}}, \ldots, d_{i_{k}}\right)\right] \cdot \mathbf{f}^{(j)}\left[d_{i_{1}}, \ldots, d_{i_{k}}\right]=\mathbf{f}^{(j)}\left[d_{i_{1}}, \ldots, d_{i_{k}}\right] .
$$

The reader should be warned here that the mappings

$$
\mathbf{e}: D \longrightarrow R(\mathcal{V},|D|)
$$

and

$$
\mathbf{f}^{(j)}: D^{k} \longrightarrow R(\mathcal{V},|D|)
$$

depend on the order in which the elements of $\mathbf{D}$ are listed, but once a function $\mathbf{e}$ is chosen as a bijection between $D$ and the set $\left\{e_{0}, \ldots, e_{n-1}\right\}$ (which is an orthogonal decomposition of the unit of the ring $R(\mathcal{V},|D|)$ into idempotents) then this enumeration of elements of $D$ is fixed and then used in the definition of the $\mathbf{f}^{(j)}$ 's. Thus we may assume that with every finite algebra $\mathbf{D}$ from $\mathcal{V}$ we are given mappings $\mathbf{e}, \mathbf{f}^{(j)}$ that satisfy

$$
\mathbf{e}\left[\mathbf{f}\left(d_{1}, \ldots, d_{k}\right)\right] \cdot \mathbf{f}^{(j)}\left[d_{1}, \ldots, d_{k}\right]=\mathbf{f}^{(j)}\left[d_{1}, \ldots, d_{k}\right]
$$

Now we ready to give our second definition.

Definition 2. Let $\mathbf{D}$ be a finite algebra from a congruence modular variety $\mathcal{V}$. Assume that the mappings

$$
\mathbf{e}: D \longrightarrow R(\mathcal{V},|D|)
$$

and

$$
\mathbf{f}^{(j)}: D^{k} \longrightarrow R(\mathcal{V},|D|)
$$

are chosen as in Definition 1. 
By a $\mathbf{D}$-expanded $R(\mathcal{V},|D|)$-module we mean any $R(\mathcal{V},|D|)$-module $M$ expanded by all constants of the form $\mathbf{f}^{(M)}\left(d_{1}, \ldots, d_{k}\right)$, (with $\mathbf{f}$ being a $k$-ary fundamental operation and $\left.\left(d_{1}, \ldots, d_{k}\right) \in D^{k}\right)$ such that $M$ satisfies the identities

$$
\mathbf{e}\left[\mathbf{f}\left(d_{1}, \ldots, d_{k}\right)\right] \cdot \mathbf{f}^{(M)}\left(d_{1}, \ldots, d_{k}\right)=\mathbf{f}^{(M)}\left(d_{1}, \ldots, d_{k}\right) .
$$

Given a D-expanded $R(\mathcal{V},|D|)$-module $M$ we observe that the set

$$
D[M]=\{(d, \mathbf{m}) \in D \times M: \mathbf{e}[d] \cdot \mathbf{m}=\mathbf{m}\}
$$

is closed under the operations defined by

$$
\begin{aligned}
\mathbf{f}\left(\left(d^{1}, \mathbf{m}^{1}\right), \ldots,\left(d^{k}, \mathbf{m}^{k}\right)\right) \\
\quad=\left(\mathbf{f}\left(d^{1}, \ldots, d^{k}\right), \sum_{j=1}^{k} \mathbf{f}^{(j)}\left[d^{1}, \ldots, d^{k}\right] \cdot \mathbf{m}^{j}+\mathbf{f}^{(M)}\left(d^{1}, \ldots, d^{k}\right)\right) .
\end{aligned}
$$

Put $\mathbf{D}[M]$ to be the algebra (of the same type as $\mathbf{D}$ ) with the universe $D[M]$ and the operations just defined.

We summarize our considerations in the following

Lemma 3. Let $\mathbf{D}$ be a finite algebra from a congruence modular variety $\mathcal{V}$. Assume we are given mappings $\mathbf{e}$ and $\mathbf{f}^{(j)}$ like in Definition 1 . Then every finite algebra $\mathbf{A} \in \mathcal{V}$ having an Abelian congruence $\beta$ with $\mathbf{A} / \beta \simeq \mathbf{D}$ is isomorphic to an algebra of the form $\mathbf{D}[M]$, for some $\mathbf{D}$-expanded $R(\mathcal{V},|D|)$-module $M$.

Proof. Let $\iota: \mathbf{A} \longrightarrow \mathbf{D}$ be a surjective homomorphism with kernel $\beta$. Assume that $n=|D|=|A / \beta|$ and choose $c_{0}, \ldots, c_{n-1} \in A$ such that $\mathbf{e}\left(\iota\left(c_{i}\right)\right)=e_{i}$. Then we have $M_{\mathbf{A}}(\beta)=M_{\mathbf{A}}\left(\beta, c_{0}\right) \times \ldots \times M_{\mathbf{A}}\left(\beta, c_{n-1}\right)$. By $\bar{M}_{\mathbf{A}}(\beta)$ denote the module $M_{\mathbf{A}}(\beta)$ that is $\mathbf{D}$-expanded by

$$
\mathbf{f}^{(M)}\left(\iota\left(a_{1}\right), \ldots, \iota\left(a_{k}\right)\right)=\left(u_{0}, \ldots, u_{n-1}\right)
$$

with

$$
u_{p}= \begin{cases}\mathbf{f}\left(a_{1}, \ldots, a_{k}\right) & \text { if } \mathbf{f}\left(a_{1}, \ldots, a_{k}\right) \in c_{p} / \beta \\ c_{p} & \text { otherwise. }\end{cases}
$$

Note here that this definition does not depend on the choice of the $a_{i}$ 's and that the condition $(\star \star)$ is fulfilled.

Representing each element $a$ of $A$ in the module $M_{\mathbf{A}}(\beta)$ by a tuple $\widehat{a}=\left(\widehat{a}_{0}, \ldots\right.$, $\left.\widehat{a}_{n-1}\right)$ with

we can define a mapping

$$
\widehat{a}_{i}= \begin{cases}a & \text { if } a \in c_{i} / \beta \\ c_{i} & \text { otherwise }\end{cases}
$$

$$
A \ni a \longmapsto(\iota(a), \widehat{a}) \in D\left[\bar{M}_{\mathbf{A}}(\beta)\right] .
$$

One can easily check that this mapping is bijective. Also a straightforward, but laborious, calculation shows that it is a homomorphism between $\mathbf{A}$ and $\mathbf{D}\left[\bar{M}_{\mathbf{A}}(\beta)\right]$.

Note here that the module $\bar{M}_{\mathbf{A}}(\beta)$ is uniquely, up to isomorphism, determined by the variety $\mathcal{V}$, the algebra $\mathbf{A} \in \mathcal{V}$ and the Abelian congruence congruence $\beta$ of $\mathbf{A}$. This means that different numberings of the elements of $\mathbf{A} / \beta$ (in particular resulting from different homomorphisms of $\mathbf{A}$ onto $\mathbf{A} / \beta$ ) lead to isomorphic $\mathbf{D}$ expanded $R(\mathcal{V},|A / \beta|)$-modules.

We will also need the following 
Definition 4. Assume that $\mathcal{V}$ is a finitely generated variety satisfying the conditions (m.1) $-(\mathbf{m} . \mathbf{3})$, and let $\mathbf{D} \in \mathcal{V}$ be a trivial or a subdirectly irreducible algebra with non-Abelian monolith.

Define $\mathcal{V} * \mathbf{D}$ to be the subvariety of $\mathcal{V}$ generated by all subdirectly irreducible algebras from $\mathcal{V}$ having $\mathbf{D}$ as a homomorphic image. In particular if $\mathbf{D}$ is trivial then $\mathcal{V} * \mathbf{D}=\mathcal{V}$.

By $R_{\mathbf{D}}$ we denote the ring $R(\mathcal{V} * \mathbf{D},|D|)$. The elements of the form $\mathbf{e}[d]$ and $\mathbf{f}^{(j)}\left[d_{1}, \ldots, d_{k}\right]$ are fixed as in Definition 1.

Finally, let $\mathcal{V}_{\mathbf{D}}$ be the variety of $\mathbf{D}$-expanded $R_{\mathbf{D}}$-modules generated by $\mathbf{D}$ expanded modules of the form $\bar{M}_{\mathbf{A}}(\rho)$ (described in the proof of Lemma 3), where $\mathbf{A} \in \mathcal{V}$ is a subdirectly irreducible algebra, $\rho$ is its largest solvable (=Abelian) congruence and $\mathbf{A} / \rho \simeq \mathbf{D}$.

We will need the following refinement of Lemma 3 .

Theorem 5. Assume that $\mathcal{V}$ is a finitely generated variety satisfying the conditions (m.1)-(m.3), and let $\mathbf{D} \in \mathcal{V}$ be a trivial or a subdirectly irreducible algebra with non-Abelian monolith. If $\mathbf{A} \in \mathcal{V}$ is a finite algebra with largest solvable congruence $\rho$ that is comparable with all congruences of $\mathbf{A}$ and such that $\mathbf{A} / \rho \simeq \mathbf{D}$ then $\mathbf{A} \simeq$ $\mathbf{D}[M]$ for some $\mathbf{D}$-expanded module $M$ from $\mathcal{V}_{\mathbf{D}}$. Conversely, for $M \in \mathcal{V}_{\mathbf{D}}$ we have $\mathbf{D}[M] \in \mathcal{V} * \mathbf{D}$. Moreover, if $\rho$ is the largest solvable congruence of $\mathbf{D}[M]$, then $\mathbf{D}[M] / \rho \simeq \mathbf{D}$ and $\bar{M}_{\mathbf{D}[M]}(\rho) \simeq M$.

Proof. First note that if $\mathbf{A}$ satisfies the assumptions of our theorem, then $\mathbf{A} \in \mathcal{V} * \mathbf{D}$. Indeed, if $\varphi$ is a minimal meet-irreducible congruence of $\mathbf{A}$ then $\varphi \subseteq \rho$. Thus $\mathbf{A} / \varphi$ is a subdirectly irreducible (or trivial) algebra that has $\mathbf{D}$ as a homomorphic image and therefore $\mathbf{A} / \varphi \in \mathcal{V} * \mathbf{D}$. Consequently $\mathbf{A} \in \mathcal{V} * \mathbf{D}$ as a subdirect product of subdirectly irreducibles from $\mathcal{V} * \mathbf{D}$.

Moreover, by Lemma $3, \mathbf{A} \simeq D\left[\bar{M}_{\mathbf{A}}(\rho)\right]$. We need to show that $\bar{M}_{\mathbf{A}}(\rho) \in \mathcal{V}_{\mathbf{D}}$. Obviously, for each minimal meet-irreducible congruence $\varphi$ of $\mathbf{A}$ the congruence $\rho / \varphi$ is the largest solvable congruence of $\mathbf{A} / \varphi$. By (m.3.1) and (m.3.2), each solvable congruence in any algebra from $\mathcal{V}$ is Abelian. Therefore we can form an $R_{\mathbf{D}^{-}}$module $M_{\mathbf{A} / \varphi}(\rho / \varphi)$ and, since $\mathbf{A} / \varphi$ is subdirectly irreducible, then by the definition of $\mathcal{V}_{\mathbf{D}}$, we know that a $\mathbf{D}$-expanded $R_{\mathbf{D}^{-} \text {module }} \bar{M}_{\mathbf{A} / \varphi}(\rho / \varphi)$ belongs to $\mathcal{V}_{\mathbf{D}}$. Thus it suffices to show that $\bar{M}_{\mathbf{A}}(\rho)$ is (isomorphic to) a subdirect product of the $\bar{M}_{\mathbf{A} / \varphi}(\rho / \varphi)$ 's. It should be clear that the mapping $\mu_{\varphi}$ that sends a vector $\left(u_{0}, \ldots, u_{n-1}\right)$ of $M_{\mathbf{A}}(\rho)$ to $\left(u_{0} / \varphi, \ldots, u_{n-1} / \varphi\right) \in M_{\mathbf{A} / \varphi}(\rho / \varphi)$ is a homomorphism of $R_{\mathbf{D}^{-}}$-modules. The remark made just after the proof of Lemma 3, makes it clear that $\bar{M}_{\mathbf{A} / \varphi}(\rho / \varphi)$ is isomorphic to the $\mathbf{D}$-expansion of $M_{\mathbf{A} / \varphi}(\rho / \varphi)$ by the constants $\mu_{\varphi}\left(\mathbf{f}^{\left(M_{\mathbf{A}}(\rho)\right)}\left(d^{1}, \ldots, d^{k}\right)\right)$ 's. Consequently $\mu_{\varphi}$ can be treated as a homomorphism of $\mathbf{D}$-expanded $R_{\mathbf{D}^{-}}$-modules. Now, since the $\mu_{\varphi}$ 's separate points of $M_{\mathbf{A}}(\rho)$ then we can finish the proof of the first part of theorem.

To see the second part denote by $\mathcal{K}$ the set of generators of $\mathcal{V}_{\mathbf{D}}$ described in the definition of $\mathcal{V}_{\mathbf{D}}$. Now note that each $\mathbf{D}$-expanded module from $\mathcal{V}_{\mathbf{D}}$ is a homomorphic image of a subdirect product of $\mathbf{D}$-expanded modules from $\mathcal{K}$. Directly from the definition of $\mathcal{K}$ and the proof of Lemma 3 we know that $\mathbf{D}[M]$ belongs to $\mathcal{V} * \mathbf{D}$ for each $M \in \mathcal{K}$.

Now, let $M$ be a subdirect product of some $\mathbf{D}$-expanded modules $M_{i}, \quad(i \in I)$ from $\mathcal{K}$. We will show that $\mathbf{D}[M]$ is a subdirect product of the $\mathbf{D}\left[M_{i}\right]$ 's. The 
subdirect embedding is given by

$$
D[M] \ni\left(d,\left(m_{i}\right)_{i \in I}\right) \longmapsto\left(\left(d, m_{i}\right)\right)_{i \in I} \in \prod_{i \in I} D\left[M_{i}\right] .
$$

To establish the last claim we only need to check that all projections of the form $D[M] \longrightarrow D\left[M_{i}\right]$ are surjective. This means that given $(d, m) \in D\left[M_{i}\right]$ there is $\mathbf{m}=\left(m_{i}\right)_{i \in I} \in M$ such that $(d, \mathbf{m}) \in D[M]$ and $m_{i}=m$. Since $M$ is a subdirect product of the $M_{i}$ 's then there is $\mathbf{n} \in M$ with $n_{i}=m$. Put $\mathbf{m}=\mathbf{e}[d] \cdot \mathbf{n}$, and observe, using $(d, m) \in D\left[M_{i}\right]$, that $m_{i}=(\mathbf{e}[d] \cdot \mathbf{n})_{i}=\mathbf{e}[d] \cdot n_{i}=\mathbf{e}[d] \cdot m=m$. Moreover, since $\mathbf{e}[d]$ is an idempotent of the $\operatorname{ring} R_{\mathbf{D}}$, then $\mathbf{e}[d] \cdot \mathbf{m}=\mathbf{m}$, which gives $(d, \mathbf{m}) \in D[M]$, as required. Consequently, $\mathbf{D}[M]$ is a subdirect product of the $\mathbf{D}\left[M_{i}\right]$ 's from $\mathcal{V} * \mathbf{D}$ and thus $\mathbf{D}[M]$ belongs to $\mathcal{V} * \mathbf{D}$.

Finally, let $M, N \in \mathcal{V}_{\mathbf{D}}$ be such that $\mathbf{D}[M] \in \mathcal{V} * \mathbf{D}$ and let $h: M \longrightarrow N$ be a surjective homomorphism of $\mathbf{D}$-expanded $R_{\mathbf{D}}$-modules. Then the mapping

$$
\bar{h}: D[M] \ni(d, m) \longmapsto(d, h(m)) \in D[N]
$$

is a surjective homomorphism and therefore $\mathbf{D}[N] \in \mathcal{V} * \mathbf{D}$.

Before proving the last part we need some preparations.

Let $D=\left\{d_{1}, \ldots, d_{s}\right\}$.

For each $\left(k\right.$-ary) term $\mathbf{t}$ of $\mathcal{V}$, using elements $\mathbf{f}^{(j)}\left[d_{i_{1}}, \ldots, d_{i_{l}}\right] \in R_{\mathbf{D}}$ and constants $\mathbf{f}^{(M)}\left(d_{i_{1}}, \ldots, d_{i_{l}}\right)$ of the language of $\mathbf{D}$-expanded $R_{\mathbf{D}}$-modules, we can compute $\mathbf{t}^{(j)}\left[d_{i_{1}}, \ldots, d_{i_{k}}\right] \in R_{\mathbf{D}}$ and constant terms $\mathbf{t}^{(M)}\left(d_{i_{1}}, \ldots, d_{i_{k}}\right)$ (in the language of $\left.\mathcal{V}_{\mathbf{D}}\right)$ such that for any $\mathbf{A} \in \mathcal{V} * \mathbf{D}$ and an Abelian congruence $\beta$ of $\mathbf{A}$ with $\mathbf{A} / \beta \simeq \mathbf{D}$ we have

$$
\mathbf{t}\left(a_{1}, \ldots, a_{k}\right)=\sum_{j=1}^{k} \mathbf{t}^{(j)}\left[d_{i_{1}}, \ldots, d_{i_{k}}\right] \cdot a_{j}+\mathbf{t}^{\left(\bar{M}_{\mathbf{A}}(\beta)\right)}\left(d_{i_{1}}, \ldots, d_{i_{k}}\right)
$$

whenever $a_{1} \in d_{i_{1}} / \beta, \ldots, a_{k} \in d_{i_{k}} / \beta$.

Assume now that $M \in \mathcal{V}_{\mathbf{D}}$ and $\rho$ is the largest solvable congruence of $\mathbf{D}[M]$.

First we want to show that $\mathbf{D}[M] / \rho \simeq \mathbf{D}$. Directly from the definition of the operations of $\mathbf{D}[M]$ we know that the mapping

$$
D[M] \ni(d, m) \longmapsto d \in D
$$

is a homomorphism. Since it is surjective it suffices to show that $\rho$ is its kernel. As $\mathbf{D}$ is trivial or subdirectly irreducible with a non-Abelian monolith then $\rho$ is contained in this kernel. Moreover, it should not be difficult to check that the definition of the fundamental operations of $\mathbf{D}[M]$ extends to arbitrary terms, i.e. in $\mathbf{D}[M]$ we have

$$
\begin{aligned}
& \mathbf{t}\left(\left(d^{1}, \mathbf{m}^{1}\right), \ldots,\left(d^{k}, \mathbf{m}^{k}\right)\right) \\
& \quad=\left(\mathbf{t}\left(d^{1}, \ldots, d^{k}\right), \sum_{j=1}^{k} \mathbf{t}^{(j)}\left[d^{1}, \ldots, d^{k}\right] \cdot \mathbf{m}^{j}+\mathbf{t}^{(M)}\left(d^{1}, \ldots, d^{k}\right)\right) .
\end{aligned}
$$

This allows us to claim that the considered kernel satisfies the term condition and therefore it is an Abelian congruence of $\mathbf{D}[M]$. Consequently it is contained in $\rho$.

It remains to show that $\bar{M}_{\mathbf{D}[M]}(\rho) \simeq M$. Consider the mapping

$$
h: M \ni m \longmapsto\left(\left(d_{1}, \mathbf{e}\left(d_{1}\right) \cdot m\right), \ldots,\left(d_{s}, \mathbf{e}\left(d_{s}\right) \cdot m\right)\right) \in M_{\mathbf{D}[M]}(\rho) .
$$

Since the $\mathbf{e}\left[d_{i}\right]$ 's are idempotent, $h$ is well defined. Using the fact that the $\mathbf{e}\left[d_{i}\right]$ 's are pairwise orthogonal and sum to the unit of $R_{\mathbf{D}}$ we get that $h$ is bijective. It 
should be obvious that $h$ preserves module addition. The proof that it preserves multiplication by scalars from the ring $R_{\mathbf{D}}$ needs more effort. First we will show that certain identities holds in $\mathcal{V}_{\mathbf{D}}$.

Assume that $\mathbf{t}=\mathbf{t}\left(v_{0}, v_{1}, \ldots, v_{s}\right) \in v_{p} / \theta_{q}$, i.e. $\mathcal{V}$ satisfies the identity

$$
\mathbf{t}\left(v_{q}, v_{1}, \ldots, v_{s}\right)=v_{p} .
$$

Let $\bar{M}_{\mathbf{A}}(\beta)$ be a generator of the variety $\mathcal{V}_{\mathbf{D}}$. Then in particular we assume that there is a surjective homomorphism $\iota: \mathbf{A} \longrightarrow \mathbf{D}$ with kernel $\beta$. Let $M_{\mathbf{A}}(\beta)$ be represented as a product $M_{\mathbf{A}}\left(\beta, a_{1}\right) \times \ldots \times M_{\mathbf{A}}\left(\beta, a_{s}\right)$ and $\iota\left(a_{i}\right)=d_{i}$ for all $i=$ $1, \ldots, s$. Since $\mathbf{t}\left(d_{q}, d_{1}, \ldots, d_{s}\right)=d_{p}$ then $\mathbf{t}^{(M)}\left(d_{q}, d_{1}, \ldots, d_{s}\right)=\left(a_{1}, \ldots, a_{s}\right)$ is the zero element of the module $M_{\mathbf{A}}(\beta)$. Therefore for each $\mathbf{t} \in v_{p} / \theta_{q}$ the equation

$$
\mathbf{t}^{(M)}\left(d_{q}, d_{1}, \ldots, d_{s}\right)=0
$$

holds in $\mathcal{V}_{\mathbf{D}}$.

Now for a vector $m=\left(m_{1}, \ldots, m_{s}\right) \in M_{\mathbf{A}}(\beta)$ with at most one non-zero entry $m_{q}$, (i.e. $m_{j}=a_{j}$ for $j \neq q$ ) we have

$$
\mathbf{t}\left(m_{q}, a_{1}, \ldots, a_{s}\right)=\left(\mathbf{t}^{(0)}\left[d_{q}, d_{1}, \ldots, d_{s}\right] \cdot m\right)_{p} .
$$

This gives that for all $m \in M_{\mathbf{A}}(\beta)$ the vector $\mathbf{t}^{(0)}\left[d_{q}, d_{1}, \ldots, d_{s}\right] \cdot \mathbf{e}\left[d_{q}\right] \cdot m$ has at most one non-zero coordinate that is equal to $\mathbf{t}\left(m_{q}, a_{1}, \ldots, a_{s}\right)$ and occurs in the $p$-th position.

On the other hand, if $\overline{\mathbf{t}}$ denotes the matrix in $R_{\mathbf{D}}$ whose $(p, q)$-th entry is $\mathbf{t} /\left[\theta_{q}, \theta_{q}\right]$ and whose other entries are zero, then

$$
\overline{\mathbf{t}} \cdot m=\left(\sum_{j=1}^{s}(\overline{\mathbf{t}})_{1 j} \cdot m_{j}, \ldots, \sum_{j=1}^{s}(\overline{\mathbf{t}})_{s j} \cdot m_{j}\right)
$$

is the vector with at most one non-zero entry (in $p$-th position) which is equal to $(\overline{\mathbf{t}})_{p q} \cdot m_{q}=\left(\mathbf{t} /\left[\theta_{q}, \theta_{q}\right]\right) \cdot m_{q}=\mathbf{t}\left(m_{q}, a_{1}, \ldots, a_{s}\right)$. Thus we have shown that $M_{\mathbf{A}}(\beta)$, and therefore the variety $\mathcal{V}_{\mathbf{D}}$, satisfies the identity

$$
\overline{\mathbf{t}} \cdot x=\mathbf{t}^{(0)}\left[d_{q}, d_{1}, \ldots, d_{s}\right] \cdot \mathbf{e}\left[d_{q}\right] \cdot x
$$

whenever $\mathbf{t} \in v_{p} / \theta_{q}$.

Now we are ready to return to the proof that $h(r \cdot m)=r \cdot h(m)$. Since $h$ is additive and $r=\sum_{i, j=1}^{s} \mathbf{e}\left[d_{i}\right] \cdot r \cdot \mathbf{e}\left[d_{j}\right]$ then it suffices to consider matrices $r$ with at most one non-zero entry, that is matrices of the form $r=\overline{\mathbf{t}}$ for some $p, q=1, \ldots, s$ and $\mathbf{t} \in v_{p} / \theta_{q}$. Since $\overline{\mathbf{t}}=\mathbf{e}\left[d_{p}\right] \cdot \overline{\mathbf{t}}$, the element $\mathbf{e}\left[d_{p}\right]$ is idempotent and $\mathbf{e}\left[d_{i}\right]$ 's are pairwise orthogonal then

$$
h(\overline{\mathbf{t}} \cdot m)=\left(\left(d_{1}, 0\right), \ldots,\left(d_{p}, \overline{\mathbf{t}} \cdot m\right), \ldots,\left(d_{s}, 0\right)\right) .
$$

On the other hand $\overline{\mathbf{t}} \cdot h(m)$ is a vector with zeros in all coordinates but the $p$-th, where $\left(\mathbf{t} /\left[\theta_{q}, \theta_{q}\right]\right) \cdot\left(d_{q}, \mathbf{e}\left[d_{q}\right] \cdot m\right)$ occurs. However,

$$
\begin{aligned}
& \left(\mathbf{t} /\left[\theta_{q}, \theta_{q}\right]\right) \cdot\left(d_{q}, \mathbf{e}\left[d_{q}\right] \cdot m\right)=\mathbf{t}\left(\left(d_{q}, \mathbf{e}\left[d_{q}\right] \cdot m\right),\left(d_{1}, 0\right), \ldots,\left(d_{s}, 0\right)\right) \\
& \quad=\left(\mathbf{t}\left(d_{q}, d_{1}, \ldots, d_{s}\right), \mathbf{t}^{(0)}\left[d_{q}, d_{1}, \ldots, d_{s}\right] \cdot \mathbf{e}\left[d_{q}\right] \cdot m+\mathbf{t}^{(M)}\left(d_{q}, d_{1}, \ldots, d_{s}\right)\right)
\end{aligned}
$$

which, in view of $\mathbf{t} \in v_{p} / \theta_{q}$ and the identities that were previously shown to hold in $\mathcal{V}_{\mathbf{D}}$, is equal to $\left(d_{p}, \overline{\mathbf{t}} \cdot m\right)$. Consequently $\overline{\mathbf{t}} \cdot h(m)=h(\overline{\mathbf{t}} \cdot m)$ and therefore $h$ is a module isomorphism. 
The reader should find no difficulty in establishing that whenever

$$
d_{i_{0}}=\mathbf{f}\left(d_{i_{1}}, \ldots, d_{i_{k}}\right)
$$

and $j \neq i_{0}$ then the identities $\mathbf{e}\left[d_{i_{0}}\right] \cdot \mathbf{f}^{(M)}\left(d_{i_{1}}, \ldots, d_{i_{k}}\right)=\mathbf{f}^{(M)}\left(d_{i_{1}}, \ldots, d_{i_{k}}\right)$ and $\mathbf{e}\left[d_{j}\right] \cdot \mathbf{f}^{(M)}\left(d_{i_{1}}, \ldots, d_{i_{k}}\right)=0$ hold in $\mathcal{V}_{\mathbf{D}}$, and then checking that $h$ preserves the constants of the form $\mathbf{f}^{(M)}\left(d_{i_{1}}, \ldots, d_{i_{k}}\right)$ which is the last step in proving that $h$ is an isomorphism of $\mathbf{D}$-expanded modules.

Our next goal is to generalize the construction of $\mathbf{D}[M]$ described in Definition 2 in such a way that each finite algebra from a variety satisfying the conditions (m.1)-(m.3) can be represented by this new construction.

Definition 6. Assume that $\mathbf{D}$ belongs to a congruence modular variety $\mathcal{V}, T$ is a poset and let $\Theta: T \longrightarrow \operatorname{Con}(\mathbf{D})$ be a monotonic mapping. The symbol $\Theta$ will also be used to denote the congruence of $\mathbf{D}^{T}$ which is obtained as a product of congruences $\left\{\Theta_{t}: t \in T\right\}$. Assume moreover that for each $t \in T$ and for each fundamental operation $\mathbf{f}$ we are given mappings

$$
\begin{array}{rcc}
\mathbf{e}_{t}: & D / \Theta_{t} & \longrightarrow R\left(\mathcal{V} *\left(\mathbf{D} / \Theta_{t}\right),\left|D / \Theta_{t}\right|\right), \\
\mathbf{f}_{t}^{(j)}:\left(D / \Theta_{t}\right)^{k} & \longrightarrow R\left(\mathcal{V} *\left(\mathbf{D} / \Theta_{t}\right),\left|D / \Theta_{t}\right|\right),
\end{array}
$$

satisfying the conditions of the Definition 1 (under some, previously chosen, numbering of the elements of $D$ ).

By a $(\mathcal{V}, \mathbf{D}, T, \Theta)$-admissible family of modules we mean a family $M=\left\{M_{t}\right.$ : $t \in T\}$, in which each $M_{t}$ is a $\left(\mathbf{D} / \Theta_{t}\right)$-expanded $R\left(\mathcal{V} *\left(\mathbf{D} / \Theta_{t}\right),\left|D / \Theta_{t}\right|\right)-$ module.

For each $(\mathcal{V}, \mathbf{D}, T, \Theta)$-admissible family $M$ of modules the set $D[T, \Theta, M]$ consisting of all elements

$$
\left(\left(d_{t} / \Theta_{t}, m_{t}\right)\right)_{t \in T} \in \prod_{t \in T}\left(D / \Theta_{t}\right)\left[M_{t}\right]
$$

satisfying:

(0) for each $d \in D$ the set $\left\{t \in T:\left(d_{t}, d\right) \in \Theta_{t}\right\}$ is closed upward, is a subuniverse of the product

$$
\prod_{t \in T}\left(\mathbf{D} / \Theta_{t}\right)\left[M_{t}\right]
$$

Thus in the corresponding algebra $\mathbf{D}[T, \Theta, M]$ the operations are given by

$$
\begin{aligned}
& \mathbf{f}\left(\left(d_{t}^{1} / \Theta_{t}, \mathbf{m}_{t}^{1}\right)_{t \in T}, \ldots,\left(d_{t}^{k} / \Theta_{t}, \mathbf{m}_{t}^{k}\right)_{t \in T}\right)=\left(\mathbf{f}\left(d_{t}^{1} \ldots d_{t}^{k}\right) / \Theta_{t},\right. \\
&\left.\sum_{j=1}^{k} \mathbf{f}_{t}^{(j)}\left[d_{t}^{1} / \Theta_{t}, \ldots, d_{t}^{k} / \Theta_{t}\right] \cdot \mathbf{m}_{t}^{j}+\mathbf{f}_{t}^{(M)}\left(d_{t}^{1} / \Theta_{t}, \ldots, d_{t}^{k} / \Theta_{t}\right)\right)_{t \in T},
\end{aligned}
$$

where the element $\mathbf{f}_{t}^{(M)}\left(d_{t}^{1} / \Theta_{t}, \ldots, d_{t}^{k} / \Theta_{t}\right)$ is the interpretation of the constant $\mathbf{f}^{(M)}\left(d_{t}^{1}, \ldots, d_{t}^{k}\right)$ in the $\mathbf{D} / \Theta_{t}$-expanded module $M_{t}$.

Since there is a natural bijection between the sets $\prod_{t \in T}\left(D_{t} / \Theta_{t} \times M_{t}\right)$ and $D^{T} / \Theta \times \prod_{t \in T} M_{t}$ we will identify $D[T, \Theta, M]$ with its image under this bijection, i.e. with the set of all pairs

$$
(\alpha / \Theta, \mathbf{m}) \in D^{T} / \Theta \times \prod_{t \in T} M_{t}
$$


satisfying

(1) for each $d \in D$ the set $\llbracket \alpha \stackrel{\Theta}{\equiv} d \rrbracket=\left\{t \in T:\left(\alpha_{t}, d\right) \in \Theta_{t}\right\}$ is closed upward,

(2) $\left(\alpha_{t} / \Theta_{t}, \mathbf{m}_{t}\right) \in\left(D / \Theta_{t}\right)\left[M_{t}\right]$ for all $t \in T$,

The above construction is actually a generalization of the so-called reduced subpower introduced by the author in [10], where (implicitly) only the situation with trivial modules was considered. The reader is referred there for the hint of the proof that $D[T, \Theta, M]$ is a subuniverse of $\prod_{t \in T}\left(\mathbf{D} / \Theta_{t}\right)\left[M_{t}\right]$ and that the condition (0) is equivalent to

$\left(0^{\prime}\right)$ if $t, s \in T$ are such that $t \leq s$ then $\left(d_{t}, d_{s}\right) \in \Theta_{s}$.

In section 5 we will show that all finite algebras from a variety $\mathcal{V}$ satisfying the conditions (m.1) $-(\mathbf{m} . \mathbf{3})$ can be represented as $\mathbf{D}[T, \Theta, M]$ where $\mathbf{D}$ is a fixed finite algebra from $\mathcal{V}, T$ ranges over finite dual trees and $M$ over $T$-indexed families of $\left(\mathbf{D} / \Theta_{t}\right)$-expanded $R_{\mathbf{D} / \Theta_{t}}$-modules from $\mathcal{V}_{\mathbf{D} / \Theta_{t}}$.

The next section is devoted to a reduction of the decidability of the class

$$
\left\{\mathbf{D}[T, \Theta, M]: T \in \mathcal{T} \text { and } M_{t} \in \mathcal{M}\right\}
$$

to the decidability of the monadic second order theory of posets from $\mathcal{T}$ and the decidability of the first order theory of modules from $\mathcal{M}$.

\section{The Translation Theorem}

In this section we are going to describe a translation of a given first order algebraic language of finite type into a monadic second order language of posets with a distinguished sequence of subsets. Our present translation is a generalization of one described in [10].

First of all, for any algebra $\mathbf{D}$ we define a monadic second order language $\mathcal{L} * \mathbf{D}$, which consists of a binary predicate symbol $\leq$ and a sequence $T_{a, b}$, with $a, b \in D$, of unary predicate symbols treated as constants of the second order. Now, by a $\mathbf{D}$-poset we mean a structure of the language $\mathcal{L} * \mathbf{D}$, i.e. the sequence $\mathbf{T}=$ $\left(T ; \leq, T_{a, b}: a, b \in D\right)$, satisfying the following axioms:

(i) $\leq$ is a partial order on the nonempty set $T$,

(ii) $\left\{T_{a, b}: a, b \in D\right\}$ is a family of subsets of $T$ such that for every natural number $k$, every $k$-ary fundamental operation $\mathbf{f}$ of the algebra $\mathbf{D}$ and all $a, b, c, a_{1}, \ldots, a_{k}, b_{1}, \ldots, b_{k} \in D$, the following conditions hold:

(ii.0) $T_{a, b}$ is a closed upward subset of $T$,

(ii.1) $T_{a, a}=T$,

(ii.2) $T_{a, b}=T_{b, a}$,

(ii.3) $T_{a, b} \cap T_{b, c} \subseteq T_{a, c}$,

(ii.4) $T_{a_{1}, b_{1}} \cap \ldots \cap T_{a_{k}, b_{k}} \subseteq T_{\mathbf{f}\left(a_{1}, \ldots, a_{k}\right), \mathbf{f}\left(b_{1}, \ldots, b_{k}\right)}$.

It is clear that the above axioms can be expressed in the monadic second order language $\mathcal{L} * \mathbf{D}$. Thus defining a dual tree as a poset satisfying

$$
\forall x \forall y \forall z(z \leq x \& z \leq y) \Rightarrow(x \leq y \vee y \leq x),
$$

we have

Lemma 7. If $\mathbf{D}$ is a finite algebra of finite type then the class of all $\mathbf{D}$-posets is finitely axiomatizable in the language $\mathcal{L} * \mathbf{D}$. In particular the class of all finite dual $\mathbf{D}$-trees is finitely axiomatizable relative to the class of all finite dual trees. 
Next we observe that any $\mathbf{D}$-poset $\mathbf{T}$ is "the same thing" as a monotonic function of the form $\Theta: T \longrightarrow \operatorname{Con}(\mathbf{D})$.

Lemma 8. The structure $\mathbf{T}$ of the language $\mathcal{L} * \mathbf{D}$ is a $\mathbf{D}$-poset iff there is a monotonic function $\Theta: T \longrightarrow \operatorname{Con}(\mathbf{D})$ such that for every $a, b \in D$, the set $\llbracket a \stackrel{\Theta}{=} b \rrbracket$ defined as $\left\{t \in T:(a, b) \in \Theta_{t}\right\}$ is equal to $T_{a, b}$.

Proof. For a monotonic mapping $\Theta: T \longrightarrow \operatorname{Con}(\mathbf{D})$ the family

$$
\{\llbracket a \stackrel{\Theta}{\equiv} b \rrbracket: a, b \in D\}
$$

of subsets of $T$ satisfies the conditions (ii.0)-(ii.4). Conversely, for a $\mathbf{D}$-poset $\mathbf{T}$ the mapping

$$
\Theta: T \longrightarrow \operatorname{Con}(\mathbf{D})
$$

defined by

does the job.

$$
\Theta_{t}=\left\{(a, b) \in D \times D: t \in T_{a, b}\right\}
$$

With the above lemma in mind we can identify any $\mathbf{D}$-poset with a monotonic function of the form $\Theta: T \longrightarrow \operatorname{Con}(\mathbf{D})$, and vice versa. Consequently by a $(\mathcal{V}, \mathbf{D}, \mathbf{T})$-admissible family of modules we mean a $(\mathcal{V}, \mathbf{D}, T, \Theta)$-admissible family and $\mathbf{D}[\mathbf{T}, M]$ is used to denote the algebra $\mathbf{D}[T, \Theta, M]$. Although we should write all formulas about $\mathbf{D}$-posets in the language $\mathcal{L} * \mathbf{D}$, sometimes it is more convenient to use the corresponding map $\Theta$ instead of the family $\left\{T_{a, b}: a, b \in D\right\}$.

By a $\mathbf{D}$-expanded module language, $\mathcal{L}_{M} * \mathbf{D}$, we mean the first order language of $\mathbf{D}$-expanded $R_{\mathbf{D}^{-}}$-modules. In $\mathcal{L}_{M} * \mathbf{D}$ we have in particular unary functional symbols $\mathbf{e}[d], \mathbf{f}^{(j)}\left[d_{1}, \ldots, d_{k}\right]$, as well as constants $\mathbf{f}^{(M)}\left(d_{1}, \ldots, d_{k}\right)$, for all $k$-ary fundamental operations f $\mathbf{f}$, all $j=1, \ldots, k$ and all elements $d, d_{1}, \ldots, d_{k} \in D$.

Now, if $\varphi=\varphi\left(x_{1}, \ldots, x_{k}\right)$ is a $\mathbf{D}$-expanded module formula and $M$ a $(\mathcal{V}, \mathbf{D}, \mathbf{T})-$ admissible family of modules then for $t \in T$ by $\varphi_{t}$ we mean a first order formula in the language of $\left(\mathbf{D} / \Theta_{t}\right)$-expanded $R\left(\mathcal{V} *\left(\mathbf{D} / \Theta_{t}\right),\left|D / \Theta_{t}\right|\right)$-modules with the symbols $\mathbf{e}[d], \mathbf{f}^{(j)}\left[d_{1}, \ldots, d_{k}\right]$ and $\mathbf{f}^{(M)}\left(d_{1}, \ldots, d_{k}\right)$ interpreted by $\mathbf{e}_{t}\left[d / \Theta_{t}\right]$, $\mathbf{f}_{t}^{(j)}\left[d_{1} / \Theta_{t}, \ldots, d_{k} / \Theta_{t}\right]$, and $\mathbf{f}_{t}^{(M)}\left(d_{1} / \Theta_{t}, \ldots, d_{k} / \Theta_{t}\right)$, respectively.

Moreover, for $\mathbf{m}^{1}, \ldots, \mathbf{m}^{k} \in \prod_{t \in T} M_{t}$ we put

$$
\llbracket \varphi\left[\mathbf{m}^{1}, \ldots, \mathbf{m}^{k}\right] \rrbracket=\left\{t \in T: M_{t} \models \varphi_{t}\left[\mathbf{m}_{t}^{1}, \ldots, \mathbf{m}_{t}^{k}\right]\right\} .
$$

The proof of the next lemma is left to the reader.

Lemma 9. Let $\mathbf{D}$ be a finite algebra from a congruence modular variety $\mathcal{V}$ and $D=\left\{d_{1}, \ldots, d_{m}\right\}$. Then, for every $\mathbf{D}$-poset $\mathbf{T}$, every $(\mathcal{V}, \mathbf{D}, \mathbf{T})$-admissible family $M$ of modules, every $k$-ary fundamental operation $\mathbf{f}$ of $\mathbf{D}$ and all

$$
\left(\alpha^{0} / \Theta, \mathbf{m}^{0}\right), \ldots,\left(\alpha^{k} / \Theta, \mathbf{m}^{k}\right) \in D[T, \Theta, M],
$$

we have

$$
\mathbf{D}[T, \Theta, M] \models\left(\alpha^{0} / \Theta, \mathbf{m}^{0}\right)=\mathbf{f}\left(\left(\alpha^{1} / \Theta, \mathbf{m}^{1}\right), \ldots,\left(\alpha^{k} / \Theta, \mathbf{m}^{k}\right)\right)
$$

if and only if

$$
\left.\left.\bigcap_{j=1}^{k} \llbracket \alpha^{j} \stackrel{\Theta}{=} d_{i_{j}} \rrbracket \subseteq \llbracket \mathbf{m}^{0}=\sum_{j=1}^{k} \mathbf{f}^{(j)}\left[d_{i_{1}}, \ldots, d_{i_{k}}\right] \cdot \mathbf{m}^{j}+\mathbf{f}^{(M)}\left(d_{i_{1}}, \ldots, d_{i_{k}}\right)\right]\right]
$$


for all $i_{1}, \ldots, i_{k} \in\{1, \ldots, m\}$, and

$$
\bigcap_{j=1}^{k} \llbracket \alpha^{j} \stackrel{\Theta}{=} d_{i_{j}} \rrbracket \subseteq \llbracket \alpha^{0} \stackrel{\Theta}{\equiv} d_{i_{0}} \rrbracket,
$$

whenever $i_{0}, \ldots, i_{k} \in\{1, \ldots, m\}$ are such that $\mathbf{f}\left(d_{i_{1}}, \ldots, d_{i_{k}}\right)=d_{i_{0}}$.

Now we are in a position to prove the following Feferman-Vaught like theorem.

Theorem 10. Let $\mathcal{V}$ be a congruence modular variety and $\mathbf{D} \in \mathcal{V}$ be a finite algebra with elements $d_{1}, \ldots, d_{m}$. Then there is an effective procedure which with every formula $\varphi\left(x^{0}, \ldots, x^{k}\right)$ in the language of $\mathbf{D}$ associates a companion sequence $\left(\Phi ; \varphi^{1}, \ldots, \varphi^{n}\right)$ in which

$$
\Phi=\Phi\left(U_{0,1}, \ldots, U_{0, m}, \ldots, U_{k, 1}, \ldots, U_{k, m}, V_{1}, \ldots, V_{n}\right)
$$

is a monadic second order formula of $\mathbf{D}$-posets (with free variables among the listed ones, all of which are monadic second order), and for each $l=1, \ldots, n$

$$
\varphi^{l}=\varphi^{l}\left(x^{0}, \ldots, x^{k}\right)
$$

is a $\mathbf{D}$-expanded module formula such that for any $\mathbf{D}$-poset $\mathbf{T}$, any $(\mathcal{V}, \mathbf{D}, \mathbf{T})$ admissible family $M=\left\{M_{t}: t \in T\right\}$ of modules and all

$$
\left(\alpha^{0} / \Theta, \mathbf{m}^{0}\right), \ldots,\left(\alpha^{k} / \Theta, \mathbf{m}^{k}\right) \in D[T, \Theta, M]
$$

we have

$$
\mathbf{D}[T, \Theta, M] \models \varphi\left[\left(\alpha^{0} / \Theta, \mathbf{m}^{0}\right), \ldots,\left(\alpha^{k} / \Theta, \mathbf{m}^{k}\right)\right]
$$

if and only if

$$
\mathbf{T}=\Phi\left[\ldots, U_{i, j} / \llbracket \alpha^{i} \stackrel{\Theta}{=} d_{j} \rrbracket, \ldots, V_{l} / \llbracket \varphi^{l}\left[\mathbf{m}^{0}, \ldots, \mathbf{m}^{k}\right] \rrbracket, \ldots\right] .
$$

Proof. Without loss of generality we can define the companion sequences $\left(\Phi ; \varphi^{1}\right.$, $\ldots, \varphi^{n}$ ) only for such formulas $\varphi$ which are built from atomic formulas of the form $x^{0}=x^{1}$ or $x^{0}=\mathbf{f}\left(x^{1}, \ldots, x^{k}\right)$, where $\mathbf{f}$ is a fundamental operation of $\mathbf{D}$, by using negation, disjunction and existential quantification.

For a formula $\varphi=\varphi\left(x^{0}, x^{1}\right) \equiv\left(x^{0}=x^{1}\right)$ define a companion sequence $\left(\Phi ; \varphi^{1}\right)$ by putting $\varphi^{1}\left(x^{0}, x^{1}\right) \equiv\left(x^{0}=x^{1}\right)$ and

$$
\Phi\left(U_{0,1}, \ldots, U_{0, m}, U_{1,1}, \ldots, U_{1, m}, V_{1}\right) \equiv \forall t\left(V_{1}(t) \& \bigwedge_{j=1}^{m}\left(U_{0, j}(t) \Leftrightarrow U_{1, j}(t)\right)\right) .
$$

Next, let $\varphi$ be an atomic formula $\mathbf{f}\left(x^{1}, \ldots, x^{k}\right)=x^{0}$. Then take $n=m^{k}$, and for any $\mathbf{i}=\left(i_{1}, \ldots, i_{k}\right) \in\{1, \ldots, m\}^{k}$ put

$$
\varphi^{\mathrm{i}}\left(x^{0}, \ldots, x^{k}\right) \equiv\left(x^{0}=\sum_{j=1}^{k} \mathbf{f}^{(j)}\left[d_{i_{1}}, \ldots, d_{i_{k}}\right] \cdot x^{j}+\mathbf{f}^{(M)}\left(d_{i_{1}}, \ldots, d_{i_{k}}\right)\right),
$$

while $\Phi\left(\ldots, U_{i, j}, \ldots, V_{\mathbf{i}}, \ldots\right)$ is to be defined as a conjunction of all formulas of the form

$$
\forall t\left(U_{1, i_{1}}(t) \& \ldots \& U_{k, i_{k}}(t)\right) \Rightarrow V_{\mathbf{i}}(t),
$$

where $\mathbf{i}=\left(i_{1}, \ldots, i_{k}\right)$ ranges over $\{1, \ldots, m\}^{k}$, together with the conjunction of all formulas of the form

$$
\forall t\left(U_{1, i_{1}}(t) \& \ldots \& U_{k, i_{k}}(t)\right) \Rightarrow U_{0, i_{0}}(t)
$$


with $i_{0}, \ldots, i_{k} \in\{1, \ldots, m\}$ such that $\mathbf{f}\left(d_{i_{1}}, \ldots, d_{i_{k}}\right)=d_{i_{0}}$.

If $\varphi \equiv \neg \psi$ and $\left(\Psi ; \psi^{1}, \ldots, \psi^{n}\right)$ is the companion sequence for $\psi$ then the companion sequence for $\varphi$ is defined as

$$
\left(\neg \Psi\left(\ldots, U_{i, j}, \ldots, \neg V_{l}, \ldots\right) ; \neg \psi^{1}, \ldots, \neg \psi^{n}\right) .
$$

Now, if

$$
\left(\Psi^{l}\left(\ldots, U_{i, j}, \ldots, V_{1}^{l}, \ldots, V_{n_{l}}^{l}\right) ; \psi_{1}^{l}, \ldots, \psi_{n_{l}}^{l}\right)
$$

is a companion sequence for $\psi^{l}, l=1,2$, then take

$$
\left(\Psi ; \psi_{1}^{1}, \ldots, \psi_{n_{1}}^{1}, \psi_{1}^{2}, \ldots, \psi_{n_{2}}^{2}\right)
$$

to be the companion sequence for $\varphi \equiv \psi^{1} \vee \psi^{2}$, where

$$
\Psi \equiv \Psi\left(\ldots, U_{i, j}, \ldots, V_{1}, \ldots, V_{n_{1}+n_{2}}\right)
$$

is the disjunction

$$
\Psi^{1}\left(\ldots, U_{i, j}, \ldots, V_{1}, \ldots, V_{n_{1}}\right) \vee \Psi^{2}\left(\ldots, U_{i, j}, \ldots, V_{n_{1}+1}, \ldots, V_{n_{1}+n_{2}}\right) .
$$

Finally, let $\varphi$ be the formula $\exists x^{0} \psi\left(x^{0}, x^{1}, \ldots, x^{k}\right)$ and let $\left(\Psi ; \psi^{1}, \ldots, \psi^{n}\right)$ be the companion sequence for $\psi$. Then for all $S \subseteq\{1, \ldots, n\}$ and $j=1, \ldots, m$ define a D-expanded module formula $\varphi^{S, j}\left(x^{1}, \ldots, x^{k}\right)$ to be

$$
\exists x^{0}\left(\bigwedge_{p \in S} \psi^{p}\left(x^{0}, \ldots, x^{k}\right) \& \bigwedge_{p \notin S} \neg \psi^{p}\left(x^{0}, \ldots, x^{k}\right) \quad \& \quad \mathbf{e}\left[d_{j}\right] \cdot x^{0}=x^{0}\right) .
$$

Moreover put $\Phi\left(\ldots, U_{i, j}, \ldots, V_{S, j}, \ldots\right)$ to be the formula

$$
\begin{array}{r}
\exists U_{0,1}, \ldots, U_{0, m} \exists\left\{W_{S}\right\}_{S \subseteq\{1, \ldots, n\}}\left(\operatorname{Adm}\left(U_{0,1}, \ldots, U_{0, m}\right) \& \operatorname{Part}\left\{W_{S}\right\}\right. \\
\left.\& \bigwedge_{S, j}\left(U_{0, j} \cap W_{S} \subseteq V_{S, j}\right) \& \Psi\left(V_{p} \leftarrow \bigcup_{p \in S} W_{S}\right)\right) .
\end{array}
$$

Here $\operatorname{Adm}\left(U_{1}, \ldots, U_{m}\right)$ expresses that $T$ is a join of closed upward subsets $U_{1}, \ldots, U_{m}$, which satisfy $U_{i} \cap U_{j} \subseteq T_{d_{i}, d_{j}}$, and $T_{d_{i}, d_{j}} \cap U_{j} \subseteq U_{i}$, for all $i, j=$ $1, \ldots, m$, while $\operatorname{Part}\left\{W_{S}\right\}$ is a formula asserting that the set $\left\{W_{S}\right\}_{S \subseteq\{1, \ldots, m\}}$ is a partition of $T$, and $\Psi\left(V_{p} \leftarrow \bigcup_{p \in S} W_{S}\right)$ is the formula obtained from $\Psi$ by replacing each subformula of the form $V_{p}(t)$ by the disjunction $\bigvee_{p \in S} W_{S}(t)$.

Finally put $\left(\Phi ; \ldots, \varphi^{S, j}, \ldots\right)$ to be the companion sequence for $\varphi$.

By induction one can show that the above defined sequences satisfy the condition of our theorem. To see this for atomic formulas just recall Lemma 9. The straightforward but laborious induction step is left to the reader.

From the above theorem we get

Corollary 11. Let $\mathbf{D}$ be a finite algebra from a congruence modular variety $\mathcal{V}$ of $f$ nite type and $C \subseteq \operatorname{Con}(\mathbf{D})$ be a set of congruences of $\mathbf{D}$ such that for each $\alpha \in C$ we are given a finitely decidable class $\mathcal{K}(\mathbf{D} / \alpha)$ of $(\mathbf{D} / \alpha)$-expanded $R(\mathcal{V} *(\mathbf{D} / \alpha),|D / \alpha|)$ modules. Assume moreover that $\mathcal{T}$ is a class of $\mathbf{D}$-posets with decidable monadic second order theory and such that each congruence of the form

$$
\Theta_{t}=\left\{(a, b) \in D \times D: t \in T_{a, b}\right\}, \text { with } t \in \mathbf{T} \in \mathcal{T},
$$


belongs to $C$. Let $\mathcal{D}$ be the class of all algebras of the form $\mathbf{D}[\mathbf{T}, M]$, where $M$ is a $(\mathcal{V}, \mathbf{D}, \mathbf{T})$-admissible family of finite modules with $M_{t} \in \mathcal{K}\left(\mathbf{D} / \Theta_{t}\right)$. Then the first order theory of the class $\mathcal{D}$ is decidable.

Proof. Let $\left(\Phi^{\prime} ; \varphi_{1}^{\prime}, \ldots, \varphi_{n}^{\prime}\right)$ be a companion sequence for a sentence $\varphi$ as described in Theorem 10. It is routine to write down a new companion sequence $\left(\Phi ; \varphi_{1}, \ldots\right.$, $\left.\varphi_{m}\right)$, where $m=2^{n}$, and $\varphi_{1}, \ldots \varphi_{2^{n}}$ are the various conjuncts $\bar{\varphi}_{1} \wedge \ldots \wedge \bar{\varphi}_{n}$, with $\bar{\varphi}_{i}$ being either $\varphi_{i}$ or $\neg \varphi_{i}$, and such that for any $\mathbf{D}[\mathbf{T}, M] \in \mathcal{D}$

$$
\mathbf{D}[\mathbf{T}, M] \models \varphi \quad \text { iff } \quad \mathbf{T} \models \Phi\left(\llbracket \varphi_{1} \rrbracket, \ldots, \llbracket \varphi_{m} \rrbracket\right) .
$$

Using the finite decidability of each class of the form $\mathcal{K}(\mathbf{D} / \alpha), \alpha \in C$, we can effectively compute the sets $C_{1}, \ldots, C_{m} \subseteq C$, where

$$
C_{j}=\left\{\alpha \in C: \mathcal{K}(\mathbf{D} / \alpha) \models \neg \varphi_{j}\right\} .
$$

Now, let $\operatorname{Cons}_{j}(X)$ be the following monadic second order formula in the language of D-posets:

$$
\operatorname{Cons}_{j}(X) \equiv \forall t\left(X(t) \Rightarrow \bigwedge_{\alpha \in C_{j}}\left(\bigvee_{(a, b) \in \alpha} \neg T_{a, b}(t) \quad \vee \bigvee_{(a, b) \in D \times D-\alpha} T_{a, b}(t)\right)\right)
$$

Observe that for a $\mathbf{D}$-poset $\mathbf{T}=(T, \Theta)$ and $X \subseteq T$

$$
\mathbf{T} \models \operatorname{Cons}_{j}(X) \quad \text { iff } \quad \forall t \in X\left(\Theta_{t} \notin C_{j}\right) .
$$

To decide whether $\varphi \in \operatorname{Th}(\mathcal{D})$ consider the sentence

$$
\bar{\Phi} \equiv \forall X_{1}, \ldots, X_{m}\left(\operatorname{Part}\left\{X_{1}, \ldots, X_{m}\right\} \& \bigwedge_{j=1}^{m} \operatorname{Cons}_{j}\left(X_{j}\right) \Rightarrow \Phi\left(X_{1}, \ldots, X_{m}\right)\right)
$$

for which we claim that

$$
\mathcal{D} \models \varphi \quad \text { iff } \quad \mathcal{T} \models \bar{\Phi} .
$$

To see this, first assume that $\mathcal{D} \not \models \varphi$. Then there is a $\mathbf{D}$-poset $(T, \Theta) \in \mathcal{T}$ and a $(\mathcal{V}, \mathbf{D}, T, \Theta)$-admissible family $M=\left\{M_{t}: t \in T\right\}$ of modules with $M_{t} \in \mathcal{K}\left(\mathbf{D} / \Theta_{t}\right)$ such that $\mathbf{D}[T, \Theta, M] \not \models \varphi$. By Theorem 10 we get $(T, \Theta) \not \models \Phi\left(\llbracket \varphi_{1} \rrbracket, \ldots, \llbracket \varphi_{m} \rrbracket\right)$. However the sets $\llbracket \varphi_{1} \rrbracket, \ldots, \llbracket \varphi_{m} \rrbracket$ form a partition of $T$ and from our construction of the $C_{j}$ 's we know that $(T, \Theta) \models \operatorname{Cons}_{j}\left(\llbracket \varphi_{j} \rrbracket\right)$. This means that $\bar{\Phi}$ fails to hold in $(T, \Theta)$.

To see the converse, assume that $\mathcal{D} \models \varphi$, take $(T, \Theta) \in \mathcal{T}$ and fix a partition $X_{1}, \ldots, X_{m}$ of $T$ satisfying in addition $\operatorname{Cons}_{j}\left(X_{j}\right)$ for all $j=1, \ldots, m$.

For each $t \in T$ there is a unique $j=1, \ldots, m$ with $t \in X_{j}$. However $\operatorname{Cons}_{j}\left(X_{j}\right)$ gives $\Theta_{t} \notin C_{j}$ i.e. for each $t \in T$ we can find a $\left(\mathbf{D} / \Theta_{t}\right)$-expanded module $M_{t} \in$ $\mathcal{K}\left(\mathbf{D} / \Theta_{t}\right)$ such that $M_{t} \models \varphi_{j}$.

Let $M=\left\{M_{t}: t \in T\right\}$. Our assumption $\mathcal{D} \models \varphi$ gives $\mathbf{D}[T, \Theta, M] \models \varphi$ and consequently $(T, \Theta) \models \Phi\left(\llbracket \varphi_{1} \rrbracket, \ldots, \llbracket \varphi_{m} \rrbracket\right)$. On the other hand from our construction it follows that $X_{j} \subseteq \llbracket \varphi_{j} \rrbracket$, which, together with the facts that both the $X_{j}$ 's and $\llbracket \varphi_{j} \rrbracket$ 's form partitions of $T$, gives $X_{j}=\llbracket \varphi_{j} \rrbracket$ and therefore $\Phi\left(X_{1}, \ldots, X_{m}\right)$ holds in $(T, \Theta)$. This means that $\bar{\Phi}$ holds in $\mathcal{T}$ as required. 


\section{Distributive Skeleton of Congruences}

In this section we fix a finitely generated variety $\mathcal{V}$ satisfying the conditions (m.1)-(m.3.5) of the Main theorem. We start with recalling that each finite algebra $\mathbf{A}$ has a largest solvable congruence which is to be called the solvable radical of $\mathbf{A}$ and denoted by $\operatorname{rad} \mathbf{A}$.

Lemma 12. Let $\mathbf{A}$ be a finite algebra from a congruence modular variety $\mathcal{V}$ satisfying (m.3). Then we have:

(12.1) if $\operatorname{rad} \mathbf{A}=0$ then $\operatorname{Con}(\mathbf{A})$ has no Abelian quotients,

(12.2) if $\delta \in \operatorname{Con}(\mathbf{A})$ then $\operatorname{rad}(\mathbf{A} / \delta)=(\delta \vee \operatorname{rad} \mathbf{A}) / \delta$,

(12.3) if $\delta$ is a meet irreducible congruence of $\mathbf{A}$ then $(\delta \vee \operatorname{rad} \mathbf{A}) / \delta$ is the centralizer of the monolith of the subdirectly irreducible algebra $\mathbf{A} / \delta$,

(12.4) the interval $I[\operatorname{rad} \mathbf{A}, 1]$ is a distributive sublattice of $\operatorname{Con}(\mathbf{A})$.

Proof. To see (12.1) assume to the contrary that in the variety there is a finite algebra $\mathbf{A}$ with trivial radical and an Abelian congruence quotient. Fix a minimal congruence $\alpha$ of $\mathbf{A}$ having a cover $\beta$ which is Abelian over $\alpha$. Since $\operatorname{rad} \mathbf{A}=0$ then $\alpha \neq 0$ and thus there is a subcover $\gamma \prec \alpha$. Using the minimality of $\alpha$ we know that $\alpha$ is not Abelian over $\gamma$. Choose $\varphi$ to be a maximal (meet irreducible) congruence of $\mathbf{A}$ with $\gamma \leq \varphi$ and $\alpha \not \leq \varphi$. Then $\varphi \wedge \alpha=\gamma$, which means that the interval $I[\gamma, \alpha]$ transposes up to $I[\varphi, \varphi \vee \alpha]$ and consequently the algebra $\mathbf{A} / \varphi$ has non-Abelian monolith. Therefore the centralizer of the monolith of $\mathbf{A} / \varphi$ is trivial and, by (m.3.4) and (m.3.5) there are no Abelian quotients over $\varphi$. This gives that $\varphi \wedge \beta>\gamma$ as otherwise $I[\alpha, \beta]$ transposes up to $I[\alpha \vee \varphi, \beta \vee \varphi]$ which means that the last interval is Abelian. However $(\varphi \wedge \beta) \vee \alpha$ belongs to the two element interval $I[\alpha, \beta]$ and the supposition that $(\varphi \wedge \beta) \vee \alpha=\alpha$ would give $\varphi \wedge \beta=\gamma$. Therefore $(\varphi \wedge \beta) \vee \alpha=\beta$ and thus $I[\alpha, \beta]$ transposes down to $I[\gamma, \varphi \wedge \beta]$ implying the Abelianity of the last interval. This contradicts our choice of $\alpha$ and proves (12.1).

Now note that $\operatorname{rad} \mathbf{A} \vee \delta$ is solvable over $\delta$. This proves one inequality in (12.2). To see the converse one, observe that assuming that $\operatorname{rad} \mathbf{A} \vee \delta$ has a cover which is Abelian over rad $\mathbf{A} \vee \delta$ we get an Abelian interval of congruences in the radical-free algebra $\mathbf{A} / \operatorname{rad} \mathbf{A}$, which contradicts (12.1).

The third item (12.3) follows directly from (12.2) and (m.3.1).

To see the last claim, note that if $I[\operatorname{rad} \mathbf{A}, 1]$ contains a diamond with g.l.b. $\alpha$ and l.u.b. $\beta$ then $\beta$ is Abelian over $\alpha$, which contradicts (12.1).

Now we collect some equations on congruence lattices of finite algebras from $\mathcal{V}$ which are expressible in the language of lattices endowed with an additional binary operation of commutator of congruences which, from now on, will be denoted by a juxtaposition and thus $x^{2}$ denotes $[x, x]$.

Lemma 13. The congruences of every finite algebra from $\mathcal{V}$ satisfy the following equations:

(13.1) $x \wedge y^{2}=(x \wedge y) y$,

(13.2) $x y=\left(x \wedge y^{2}\right) \vee\left(x^{2} \wedge y\right)$,

(13.3) $x y=x \wedge y \wedge(x \vee y)^{2}$,

(13.4) $x^{2} x^{2}=x^{2}$,

(13.5) $(x \vee y)^{2}=x^{2} \vee y^{2}$,

(13.6) $\left(x^{2} \vee y^{2}\right)^{2}=x^{2} \vee y^{2}$, 
(13.7) $x^{2} \wedge y^{2}=x^{2} y^{2}$

(13.8) $x^{2} \wedge y=x^{2} y$

(13.9) $\left(x^{2} \wedge y^{2}\right)^{2}=x^{2} \wedge y^{2}$,

(13.10) $(x \wedge y)^{2}=x^{2} \wedge y^{2}$,

(13.11) $(x y) z=x(y z)$,

(13.12) $(x y)^{2}=x^{2} y^{2}$.

Proof. The identity (13.1) was shown by R.Freese and R.McKenzie [7] to be equivalent (in finitely generated congruence modular varieties) to the assumption that the variety is residually small. Items (13.2) and (13.3) are equational consequences of (13.1) and modularity. The equation (13.4) means that each solvable congruence of a finite algebra from $\mathcal{V}$ is Abelian. This fact, in turn, follows easily from (m.3.1) and (m.3.2). Items (13.5)-(13.8) are clear in view of (13.1)-(13.4). Analogously (13.10)-(13.12) are equational consequences of the previous equations.

Thus to finish the proof we need to show that (13.9) holds. However in view of (13.4) we have only to prove that

$$
(x \wedge y)^{2}=x \wedge y \quad \text { whenever } \quad x^{2}=x \quad \text { and } \quad y^{2}=y .
$$

Suppose to the contrary that we have a finite algebra $\mathbf{A} \in \mathcal{V}$ and two congruences $\alpha, \beta$ of $\mathbf{A}$ such that $\alpha^{2}=\alpha, \beta^{2}=\beta$ and $(\alpha \wedge \beta)^{2}<\alpha \wedge \beta$. Take $\eta$ such that $(\alpha \wedge \beta)^{2} \leq \eta \prec \alpha \wedge \beta$ and let $\xi$ be a maximal congruence lying over $\eta$ but not over $\alpha \wedge \beta$. Obviously $\mathbf{A} / \xi$ is subdirectly irreducible with monolith $((\alpha \wedge \beta) \vee \xi) / \xi$. Since

$$
((\alpha \wedge \beta) \vee \xi)^{2}=(\alpha \wedge \beta)^{2} \vee \xi^{2} \leq \eta \vee \xi=\xi
$$

then the monolith of $\mathbf{A} / \xi$ is Abelian. Denoting by $\rho$ the largest solvable congruence of $\mathbf{A}$, which has to be Abelian, and applying (12.3) we know that the centralizer of the monolith of $\mathbf{A} / \xi$ is $\rho \vee \xi / \xi$ and therefore, by the condition (m.3.4), the congruences of $\mathbf{A}$ above $\rho \vee \xi$ form a chain. Thus without loss of generality we can assume that $\alpha \vee \rho \vee \xi \leq \beta \vee \rho \vee \xi$, which gives

$$
\alpha=\alpha^{2} \leq(\beta \vee \rho \vee \xi)^{2}=(\beta \vee \xi)^{2} \vee \rho^{2} \leq \beta \vee \xi \text {. }
$$

Now we have

$$
\alpha=\alpha^{2} \alpha \leq \alpha^{2}(\beta \vee \xi)=\alpha^{2} \beta \vee \alpha^{2} \xi=\left(\alpha^{2} \wedge \beta\right) \vee\left(\alpha^{2} \wedge \xi\right)=(\alpha \wedge \beta) \vee(\alpha \wedge \xi) \leq \alpha,
$$

i.e. $(\alpha \wedge \beta) \vee(\alpha \wedge \xi)=\alpha$, and, by the choice of $\eta$ and $\xi$,

$$
(\alpha \wedge \beta) \wedge(\alpha \wedge \xi)=\eta
$$

which means that the interval $I[\eta, \alpha \wedge \beta]$ transposes up to $I[\alpha \wedge \xi, \alpha]$. Since $\alpha \wedge \beta$ is Abelian over $\eta$ then $\alpha$ is Abelian over $\alpha \wedge \xi$ and consequently we get the following contradiction

$$
\alpha=\alpha^{2} \leq \alpha \wedge \xi<\alpha,
$$

which finishes the proof of (13.9).

In the following we need an additional binary operation $\rightarrow$ in a congruence lattice. Given an algebra $\mathbf{A}$ from a congruence modular variety and two of its congruences $\alpha, \beta$ we put $\alpha \rightarrow \beta$ to be the largest congruence $\theta$ of $\mathbf{A}$ such that $\alpha \theta \leq \beta$. The congruence $\alpha \rightarrow \beta$ is called in [7] a centralizer of $\alpha$ over $\beta$.

We will freely use the following properties of centralizers, the proofs of which are straightforward.

$$
\begin{aligned}
& \text { - }(\alpha \vee \beta) \rightarrow \gamma=(\alpha \rightarrow \gamma) \wedge(\beta \rightarrow \gamma), \\
& \text { - } \gamma \rightarrow(\alpha \wedge \beta)=(\gamma \rightarrow \alpha) \wedge(\gamma \rightarrow \beta),
\end{aligned}
$$


- $\beta \leq \alpha \rightarrow \beta$,

- $\alpha \beta \rightarrow \gamma=\alpha \rightarrow(\beta \rightarrow \gamma)$, provided the commutator multiplication is associative.

The main goal of this section is to select, for every finite algebra $\mathbf{A} \in \mathcal{V}$, a separating set of congruences that satisfy certain distributivity conditions. This set is to be used in representing $\mathbf{A}$ by the construction described in Definition 6 .

Let us start with a fixed finite algebra $\mathbf{A} \in \mathcal{V}$. By $\operatorname{Cm}(\mathbf{A})$ we denote the set of all non-unit meet irreducible congruences of $\mathbf{A}$. Let $\rho$ be the largest solvable congruence of $\mathbf{A}$, which in view of $\mathbf{( m . 3 . 1 )}$ and (m.3.2) is Abelian. Moreover, by $(\mathbf{m . 3 . 4})-(\mathbf{m} . \mathbf{3 . 5})$, we know that the interval $I[\rho, 1]$ is a distributive lattice in which the set $T$ of meet irreducible members is a dual tree. Put $T_{+}=T \cup\{1\}$. Now we see that each member $\varphi$ of $T$ has a unique cover, say $\varphi_{+}$, in $T_{+}$, which is also a unique cover in $\operatorname{Con}(\mathbf{A})$. Finally for each $\varphi \in T_{+}$we define a congruence $\bar{\varphi}$ of $\mathbf{A}$ by putting

1. $\overline{1}=1^{2}$,

2. $\bar{\varphi}=\varphi_{+}^{2} \rightarrow \varphi^{2}$, for $\varphi \in T$.

We state some properties of the family $\left\{\bar{\varphi}: \varphi \in T_{+}\right\}$in the following

Lemma 14. For each $\varphi, \varphi_{1}, \varphi_{2} \in T_{+}$the following hold:

(14.1) $\varphi^{2} \leq \bar{\varphi} \leq \varphi$

(14.2) $\bar{\varphi}=\varphi$ or $\varphi \rightarrow \bar{\varphi}=\varphi$,

(14.3) if $\varphi \neq 1$ then $\varphi_{+} \rightarrow \bar{\varphi}=\bar{\varphi}$,

(14.4) $\varphi^{2} \vee \rho=\bar{\varphi} \vee \rho=\varphi$,

(14.5) $\varphi_{1}^{2} \leq \varphi_{2}^{2} \quad$ iff $\varphi_{1} \leq \varphi_{2}$,

(14.6) if $\varphi \neq 1$ then $\bar{\varphi} \wedge \varphi_{+}^{2}=\varphi^{2}$,

(14.7) if $\varphi \neq 1$ then $\bar{\varphi} \vee \varphi_{+}^{2}=\varphi_{+}$,

(14.8) if $\varphi_{1}<\varphi_{2}$ then $\bar{\varphi}_{1} \vee \varphi_{2}^{2}=\varphi_{2}$,

(14.9) $I[\bar{\varphi}, 1]=I[\bar{\varphi}, \varphi] \cup I[\varphi, 1]$,

(14.10) if $\varphi_{1} \neq \varphi_{2}$ then $\bar{\varphi}_{1} \vee \bar{\varphi}_{2}=\varphi_{1} \vee \varphi_{2}$,

(14.12) $\bigcap_{\varphi \in T_{+}}^{\psi \geq \varphi} \bar{\varphi}=0$.

Proof. The first inequality in (14.1) follows directly from the definition. As for $\bar{\varphi} \leq \varphi$ note that it is obvious for $\varphi=1$ and for $\varphi \neq 1$ that we have $\bar{\varphi}=\varphi_{+}^{2} \rightarrow$ $\varphi^{2} \leq \varphi_{+}^{2} \rightarrow \varphi=\varphi$, where the last equality holds, as otherwise $\varphi<\varphi_{+}^{2} \rightarrow \varphi$, i.e. $\varphi_{+} \leq \varphi_{+}^{2} \rightarrow \varphi$ and consequently $\varphi_{+}$would be Abelian over $\varphi$.

Now note that (14.2) holds for $\varphi=1$. Thus assume that $\varphi \neq 1$ and $\varphi \rightarrow \bar{\varphi} \neq \varphi$, which actually means $\varphi_{+} \leq \varphi \rightarrow \bar{\varphi}$. Using (13.4) and (13.11) we get $\varphi_{+}^{2} \varphi=$ $\varphi_{+} \varphi \varphi_{+}^{2} \leq \varphi^{2}$ and consequently $\varphi \leq \bar{\varphi}$.

To see (14.3) note only that $\varphi_{+} \rightarrow \bar{\varphi}=\varphi_{+} \rightarrow\left(\varphi_{+}^{2} \rightarrow \varphi^{2}\right)=\left(\varphi_{+} \varphi_{+}^{2}\right) \rightarrow \varphi^{2}=$ $\varphi_{+}^{2} \rightarrow \varphi^{2}=\bar{\varphi}$.

As for (14.4) note that $\varphi^{2} \vee \rho \leq \bar{\varphi} \vee \rho \leq \varphi$. However, if $\varphi^{2} \vee \rho<\varphi$ then there is a meet irreducible congruence $\psi \geq \rho$ such that $\varphi^{2} \leq \psi$ and $\varphi \not \leq \psi$. This gives that $\varphi \vee \psi$ is Abelian over $\psi$, which in view of (m.3.5), means that $\varphi \vee \psi=\psi$, a contradiction.

The equivalence (14.5) is an immediate consequence of (14.4), while (14.6) follows directly from the definition of $\bar{\varphi}$ and (13.8). 
The proof of (14.7) is more laborious. Fix the smallest, with respect to cardinality, finite algebra $\mathbf{A} \in \mathcal{V}$ having a meet irreducible congruence $\varphi$ above the radical $\rho$ of $\mathbf{A}$ such that $\bar{\varphi} \vee \varphi_{+}^{2}<\varphi_{+}$. Then obviously $\bar{\varphi}=0$ and $\varphi=\rho$. Moreover $\bar{\varphi}<\varphi$, as otherwise $\bar{\varphi} \vee \varphi_{+}^{2}=\varphi \vee \varphi_{+}^{2} \geq \varphi$ and for $\varphi \vee \varphi_{+}^{2}=\varphi$ we would get that $\varphi_{+}$ is Abelian over $\varphi$. We are going to show that $\mathbf{A}$ is subdirectly irreducible. Thus assume to the contrary that $\operatorname{Con}(\mathbf{A})$ has at least two different atoms, say $\alpha_{1}, \alpha_{2}$. Obviously we have

$$
\alpha_{1} \wedge \alpha_{2}=\bar{\varphi}
$$

Moreover,

$$
\alpha_{i} \leq \varphi
$$

as otherwise $\alpha_{i} \varphi \leq \alpha_{i} \wedge \varphi=\bar{\varphi}$, i.e., by (14.2) and $\bar{\varphi}<\varphi, \alpha_{i} \leq \varphi \rightarrow \bar{\varphi}=\varphi$.

We also have

$$
\alpha_{i} \leq \varphi_{+}^{2},
$$

for if not, then $\bar{\varphi}=\alpha_{i} \wedge \varphi_{+}^{2}=\alpha_{i} \varphi_{+}^{2}$ and consequently $\alpha_{i} \leq \varphi_{+}^{2} \rightarrow \bar{\varphi}=\bar{\varphi}=0$.

Now we put $\xi_{i}=\varphi_{+}^{2} \rightarrow \alpha_{i}$ and observe that

$$
\xi_{1} \wedge \xi_{2}=\left(\varphi_{+}^{2} \rightarrow \alpha_{1}\right) \wedge\left(\varphi_{+}^{2} \rightarrow \alpha_{2}\right)=\varphi_{+}^{2} \rightarrow\left(\alpha_{1} \wedge \alpha_{2}\right)=\varphi_{+}^{2} \rightarrow \bar{\varphi}=\bar{\varphi} .
$$

Since $\xi_{i}$ corresponds to $\overline{\varphi / \alpha_{i}}$ computed in $\mathbf{A} / \alpha_{i}$ then, using $\left|A / \alpha_{i}\right|<|A|$ and the minimality of $\mathbf{A}$ we obtain

$$
\xi_{i} \vee \varphi_{+}^{2}=\varphi_{+}
$$

Consequently

$$
\alpha_{i}<\xi_{i},
$$

as otherwise, by (2) and (1), $\varphi_{+}=\alpha_{i} \vee \varphi_{+}^{2}=\varphi_{+}^{2}$, which contradicts our assumption that $\bar{\varphi} \vee \varphi_{+}^{2}<\varphi_{+}$.

Moreover $\varphi_{+}^{2} \wedge \xi_{i}=\varphi_{+}^{2} \xi_{i} \leq \alpha_{i}$, which together with (1) and (3) gives

$$
\varphi_{+}^{2} \wedge \xi_{i}=\alpha_{i}=\varphi_{+}^{2} \xi_{i} .
$$

Now we have the following two equations

$$
\varphi_{+}^{2} \wedge\left(\xi_{1} \vee \xi_{2}\right)=\varphi_{+}^{2}\left(\xi_{1} \vee \xi_{2}\right)=\varphi_{+}^{2} \xi_{1} \vee \varphi_{+}^{2} \xi_{2}=\alpha_{1} \vee \alpha_{2}
$$

and

$$
\varphi_{+}^{2} \vee\left(\alpha_{1} \vee \xi_{2}\right)=\left(\varphi_{+}^{2} \vee \xi_{2}\right) \vee \alpha_{1}=\varphi_{+} \vee \alpha_{1}=\varphi_{+},
$$

from which by modularity we infer

$$
\alpha_{1} \vee \xi_{2}=\xi_{1} \vee \xi_{2} .
$$

Once more applying modularity we get

$$
\xi_{1}=\xi_{1} \wedge\left(\alpha_{1} \vee \xi_{2}\right)=\alpha_{1} \vee\left(\xi_{1} \wedge \xi_{2}\right)=\alpha_{1} \vee \bar{\varphi}=\alpha_{1},
$$

a contradiction with (3).

Consequently $\bar{\varphi}$ has exactly one cover and therefore $\mathbf{A}$ is subdirectly irreducible. This, by (m.3.3), means that the solvable radical of $\mathbf{A}$, i.e. the congruence $\varphi$, is comparable with $\bar{\varphi} \vee \varphi_{+}^{2}$. However $\bar{\varphi} \vee \varphi_{+}^{2}<\varphi_{+}$and therefore $\bar{\varphi} \vee \varphi_{+}^{2} \leq \varphi$, which gives that $\varphi_{+}$is Abelian over $\varphi$. The obtained contradiction proves (14.7).

Now (14.8) follows from (14.7) by induction on the length of the chain $I\left[\varphi_{1}, \varphi_{2}\right]$.

To see (14.9) take $\eta \geq \bar{\varphi}$. If $\eta \not \leq \varphi$ then $\varphi_{+} \leq \varphi \vee \eta$. Consequently, $\varphi_{+}^{2} \leq \varphi^{2} \vee \eta^{2}=$ $\eta^{2} \leq \eta$ and, by (14.7), we get $\varphi_{+}=\varphi_{+}^{2} \vee \bar{\varphi} \leq \eta$. 
As to (14.10), first note that $\bar{\varphi}_{1} \vee \bar{\varphi}_{2} \geq \rho$. Indeed, since $\bar{\varphi}_{1} \vee \bar{\varphi}_{2} \geq \bar{\varphi}_{i}$ then, by (14.9), $\bar{\varphi}_{1} \vee \bar{\varphi}_{2}$ is comparable with both $\varphi_{1}$ and $\varphi_{2}$. If $\bar{\varphi}_{1} \vee \bar{\varphi}_{2} \geq \varphi_{i}$ for some $i=1,2$ then we are done. Thus suppose $\bar{\varphi}_{1} \vee \bar{\varphi}_{2} \leq \varphi_{1} \wedge \varphi_{2}$. Then $\varphi_{i}^{2} \leq \bar{\varphi}_{i} \leq \bar{\varphi}_{1} \vee \bar{\varphi}_{2} \leq \varphi_{j}$, and consequently $\varphi_{1}^{2}=\varphi_{2}^{2}$, which together with (14.5) contradicts the assumption that $\varphi_{1} \neq \varphi_{2}$. Now, using $\bar{\varphi}_{1} \vee \bar{\varphi}_{2} \geq \rho$ and (14.4), we get $\bar{\varphi}_{1} \vee \bar{\varphi}_{2}=\bar{\varphi}_{1} \vee \bar{\varphi}_{2} \vee \rho=$ $\varphi_{1} \vee \varphi_{2}$

The item (14.11) can be shown by induction on the length of the chain $I[\varphi, 1]$. The claim is trivial for $\varphi=1$, while the induction step follows easily by (14.6).

We can finish the proof of our lemma by observing that

$$
\rho=\bigcap_{\varphi \in T_{+}} \varphi
$$

which, together with (13.10) and (14.11) gives

$$
0=\rho^{2}=\bigcap_{\varphi \in T_{+}} \varphi^{2}=\bigcap_{\varphi \in T_{+}} \bar{\varphi} .
$$

We will also need

Lemma 15. Let $\varphi, \alpha \in T_{+}, \delta$ be an arbitrary meet irreducible congruence of $\mathbf{A}$ and $\rho$ be the solvable radical of $\mathbf{A}$. Then we have

(15.1) $\delta \vee \rho \in T_{+}$,

(15.2) if $\varphi \neq 1$ and $\varphi \geq \delta$ then $\varphi_{+}^{2} \rightarrow \delta=\delta$,

(15.3) if $\varphi^{2} \leq \delta \leq \varphi$ then $\bar{\varphi} \leq \delta$,

(15.4) there is $\gamma \in T_{+}$such that $\bar{\gamma} \leq \delta \leq \gamma$,

(15.5) if $\alpha^{2} \leq \delta$ then $\bar{\gamma} \leq \delta \leq \gamma$ for some $\gamma \geq \alpha$ with $\gamma \in T_{+}$,

(15.6) if $\bar{\alpha} \not \leq \delta$ then $\delta \vee \bar{\alpha}=\delta \vee \alpha$.

Proof. To see (15.1) observe that by applying (12.3) and (m.3.4) to the subdirectly irreducible algebra $\mathbf{A} / \delta$ we get that the interval $I[\delta \vee \rho, 1]$ is a finite chain. Consequently $\delta \vee \rho=1$ or $\delta \vee \rho$ is meet irreducible.

Before proving (15.2) note that for any algebra $\mathbf{B}$ from a congruence modular variety, and all congruences $\alpha, \beta, \gamma$ of $\mathbf{B}$ if $\alpha \wedge \beta \geq \gamma$ then the centralizers $\alpha \rightarrow \beta$ computed in $\operatorname{Con}(\mathbf{B})$ and in the interval $I[\gamma, 1]$ coincide.

Now, to see that (15.2) holds observe that $\delta \leq \varphi_{+} \rightarrow \delta$. Since $\varphi_{+} \geq \varphi \geq \delta$ than we can compute $\varphi_{+} \rightarrow \delta$ in the interval $I[\delta, 1]$, or, in other words, in the congruence lattice of the subdirectly irreducible algebra $\mathbf{A} / \delta$. If $\delta<\varphi_{+} \rightarrow \delta$ then the centralizer of the monolith of $\mathbf{A} / \delta$ would lie over $\varphi_{+} / \delta$ which, by (m.3.2) would give $\varphi_{+}^{2} \leq \delta \vee \rho \leq \varphi$, a contradiction with the fact that $\varphi_{+}$is not Abelian over $\varphi$. Thus $\varphi_{+} \rightarrow \delta=\delta$. Consequently $\varphi_{+}^{2} \rightarrow \delta=\varphi_{+} \rightarrow\left(\varphi_{+} \rightarrow \delta\right)=\varphi_{+} \rightarrow \delta=\delta$ and (15.2) is shown.

Now if $\varphi=1$ then $\bar{\varphi}=\varphi^{2} \leq \delta$, while for $\varphi \neq 1$ the item (15.2) gives $\bar{\varphi}=\varphi_{+}^{2} \rightarrow$ $\varphi^{2} \leq \varphi_{+}^{2} \rightarrow \delta=\delta$, which shows (15.3).

As to (15.4) and (15.5), put $\gamma=\delta \vee \rho$, which, by (15.1) belongs to $T_{+}$. Now $\gamma^{2}=\delta^{2} \leq \delta \leq \gamma$, and using (15.3) we get $\bar{\gamma} \leq \delta \leq \gamma$. Moreover, if $\alpha^{2} \leq \delta$ then $\alpha^{2} \leq \delta^{2}=\gamma^{2}$ and by (14.5) we have $\alpha \leq \gamma$.

To see (15.6) choose, using (15.4), $\gamma \in T_{+}$with $\bar{\gamma} \leq \delta$. Since $\alpha \neq \gamma$ then by (14.10) we get $\alpha \leq \alpha \vee \gamma=\bar{\alpha} \vee \bar{\gamma} \leq \bar{\alpha} \vee \delta$. 
Definition 16. A subset $S$ of a lattice $L$ is said to be distributive if

$$
a \vee\left(s_{1} \wedge \ldots \wedge s_{n}\right)=\left(a \vee s_{1}\right) \wedge \ldots \wedge\left(a \vee s_{n}\right)
$$

for all $a \in L$ and every finite subset $\left\{s_{1}, \ldots, s_{n}\right\}$ of $S$.

We are going to show that the set $\left\{\bar{\varphi}: \varphi \in T_{+}\right\}$is a distributive subset of $\operatorname{Con}(\mathbf{A})$.

Theorem 17. If $\delta$ is a meet irreducible congruence of an algebra $\mathbf{A} \in \mathcal{V}$ and $\alpha_{1}, \ldots, \alpha_{n} \in T_{+}$are such that $\bar{\alpha}_{1} \wedge \ldots \wedge \bar{\alpha}_{n} \leq \delta$ then $\bar{\alpha}_{i} \leq \delta$ for some $i=1, \ldots, n$. Consequently the subset $\left\{\bar{\varphi}: \varphi \in T_{+}\right\}$of $\operatorname{Con}(\mathbf{A})$ is distributive.

Proof. First note that the second part follows easily from the first one. Actually we have even more: in every algebraic lattice $L$ if $s_{1} \wedge \ldots \wedge s_{n} \leq d$ with meet irreducible $d \in L$ implies $s_{i} \leq d$ for some $i=1, \ldots, n$, then the subset $\left\{s_{1}, \ldots, s_{n}\right\}$ of $L$ is distributive. Indeed, we have to show that $a \vee\left(s_{1} \wedge \ldots \wedge s_{n}\right) \geq\left(a \vee s_{1}\right) \wedge \ldots \wedge\left(a \vee s_{n}\right)$. Take a meet irreducible $d$ with $d \geq a \vee\left(s_{1} \wedge \ldots \wedge s_{n}\right)$. Then $d \geq a$ and, by the assumption $d \geq s_{i}$ for some $i=1, \ldots, n$, This obviously gives $d \geq\left(a \vee s_{1}\right) \wedge \ldots \wedge$ $\left(a \vee s_{n}\right)$ and we can finish the proof of our claim by recalling that in each algebraic lattice every element is a meet of meet irreducibles.

The first part of the theorem we will prove by induction on $n$. For $n=1$ this is trivial. Now assume that $\bar{\alpha}_{1} \wedge \ldots \wedge \bar{\alpha}_{n} \leq \delta$ and that the theorem holds for any subset of $\left\{\bar{\varphi}: \varphi \in T_{+}\right\}$with at most $n-1$ elements. By our claim this means that any such subset is distributive.

Obviously we may assume that all $\alpha_{i}$ 's are distinct. In our proof we will consider two cases.

CASE 1. There is the smallest congruence in the set $\left\{\alpha_{1}, \ldots, \alpha_{n}\right\}$.

As this smallest congruence belongs to $T_{+}$then, by (m.3.4), the set $\left\{\alpha_{1}, \ldots, \alpha_{n}\right\}$ is linearly ordered and, without loss of generality, we may assume that $\alpha_{1}<\ldots<$ $\alpha_{n}$. Here we use nested induction on the number of elements in the chain $I\left[\alpha_{1}, 1\right]$. Since $\alpha_{1}^{2} \leq \bar{\alpha}_{1} \wedge \ldots \wedge \bar{\alpha}_{n} \leq \delta$ then, by (15.5), we get $\gamma \geq \alpha_{1}$ with $\bar{\gamma} \leq \delta \leq \gamma$. If the number $\left|I\left[\alpha_{1}, 1\right]\right|$ is minimal, i.e. equal to $n$, then $\gamma \in\left\{\alpha_{1}, \ldots, \alpha_{n}\right\}$ and we are done. We then assume that $\gamma \notin\left\{\alpha_{1}, \ldots, \alpha_{n}\right\}$ and consider two cases.

CASE 1.1. $\alpha_{1}<\ldots<\alpha_{n}<\gamma$.

By (14.8) for any $i=1, \ldots, n-1$ we get $\alpha_{n}=\bar{\alpha}_{i} \vee \alpha_{n}^{2} \leq \bar{\alpha}_{i} \vee\left(\bar{\alpha}_{n} \wedge \delta\right) \leq \alpha_{n}$. This together with the induction hypothesis gives $\left(\bar{\alpha}_{1} \wedge \ldots \wedge \bar{\alpha}_{n-1}\right) \vee\left(\bar{\alpha}_{n} \wedge \delta\right)=$ $\left(\bar{\alpha}_{1} \vee\left(\bar{\alpha}_{n} \wedge \delta\right)\right) \wedge \ldots \wedge\left(\bar{\alpha}_{n-1} \vee\left(\bar{\alpha}_{n} \wedge \delta\right)\right)=\alpha_{n}$. Finally, using modularity we have $\bar{\alpha}_{n}=\bar{\alpha}_{n} \wedge \alpha_{n}=\bar{\alpha}_{n} \wedge\left(\left(\bar{\alpha}_{1} \wedge \ldots \wedge \bar{\alpha}_{n-1}\right) \vee\left(\bar{\alpha}_{n} \wedge \delta\right)\right)=\left(\bar{\alpha}_{1} \wedge \ldots \wedge \bar{\alpha}_{n}\right) \vee\left(\bar{\alpha}_{n} \wedge \delta\right) \leq \delta$.

CASE 1.2. There is $j=1, \ldots, n-1$ with $\alpha_{1}<\ldots<\alpha_{j}<\gamma<\alpha_{j+1}<\ldots<\alpha_{n}$.

Define $\beta=\left(\bar{\alpha}_{1} \wedge \ldots \wedge \bar{\alpha}_{j}\right) \vee\left(\delta \wedge \bar{\alpha}_{j+1} \wedge \ldots \wedge \bar{\alpha}_{n}\right)$ and observe that $\beta \leq \gamma$. On the other hand we have $\beta \geq\left(\bar{\alpha}_{1} \wedge \ldots \wedge \bar{\alpha}_{j}\right) \vee\left(\bar{\gamma} \wedge \bar{\alpha}_{j+1} \wedge \ldots \wedge \bar{\alpha}_{n}\right)$. Now note that by the outer and inner induction hypotheses, respectively, the sets $\left\{\bar{\alpha}_{1}, \ldots, \bar{\alpha}_{j}\right\}$ and $\left\{\bar{\gamma}, \bar{\alpha}_{j+1}, \ldots, \bar{\alpha}_{n}\right\}$ are distributive. Distributing over and using (14.10) we get $\beta \geq \gamma$. Thus $\beta=\gamma$.

Now, modularity gives $\gamma \wedge \bar{\alpha}_{j+1} \wedge \ldots \wedge \bar{\alpha}_{n}=\beta \wedge\left(\gamma \wedge \bar{\alpha}_{j+1} \wedge \ldots \wedge \bar{\alpha}_{n}\right)=\left(\left(\bar{\alpha}_{1} \wedge \ldots \wedge\right.\right.$ $\left.\left.\bar{\alpha}_{j}\right) \vee\left(\delta \wedge \bar{\alpha}_{j+1} \wedge \ldots \wedge \bar{\alpha}_{n}\right)\right) \wedge\left(\gamma \wedge \bar{\alpha}_{j+1} \wedge \ldots \wedge \bar{\alpha}_{n}\right)=\left(\bar{\alpha}_{1} \wedge \ldots \wedge \bar{\alpha}_{j} \wedge \gamma \wedge \bar{\alpha}_{j+1} \wedge \ldots \wedge \bar{\alpha}_{n}\right) \vee$ $\left(\delta \wedge \bar{\alpha}_{j+1} \wedge \ldots \wedge \bar{\alpha}_{n}\right) \leq \delta$. Finally, (14.10), distributivity of the subset $\left\{\bar{\alpha}_{j+1}, \ldots, \bar{\alpha}_{n}\right\}$ and modularity of $\operatorname{Con}(\mathbf{A})$ give $\bar{\alpha}_{j} \leq \alpha_{j} \leq \gamma=\gamma \wedge\left(\left(\gamma \vee \alpha_{j+1}\right) \wedge \ldots \wedge\left(\gamma \vee \alpha_{n}\right)\right)=$ $\gamma \wedge\left(\left(\bar{\gamma} \vee \bar{\alpha}_{j+1}\right) \wedge \ldots \wedge\left(\bar{\gamma} \vee \bar{\alpha}_{n}\right)\right)=\gamma \wedge\left(\bar{\gamma} \vee\left(\bar{\alpha}_{j+1} \wedge \ldots \wedge \bar{\alpha}_{n}\right)\right)=\left(\gamma \wedge \bar{\alpha}_{j+1} \wedge \ldots \wedge \bar{\alpha}_{n}\right) \vee \bar{\gamma} \leq \delta$ as required. This finishes the proof in case 1 .

CASE 2. There is no smallest congruence in the set $\left\{\alpha_{1}, \ldots, \alpha_{n}\right\}$. 
From $\bar{\alpha}_{1} \wedge \ldots \wedge \bar{\alpha}_{n} \leq \delta$ we get $\alpha_{1}^{2} \wedge \ldots \wedge \alpha_{n}^{2} \leq \delta^{2} \leq(\delta \vee \rho)^{2}$, i.e. (by (13.5) and $(13.9))(\delta \vee \rho)^{2}=\left((\delta \vee \rho) \vee\left(\alpha_{1} \wedge \ldots \wedge \alpha_{n}\right)\right)^{2}$. Since, by (15.1), both $\delta \vee \rho$ and $(\delta \vee \rho) \vee\left(\alpha_{1} \wedge \ldots \wedge \alpha_{n}\right)$ are in $T_{+}$then, by (14.5), we get $\alpha_{1} \wedge \ldots \wedge \alpha_{n} \leq \delta \vee \rho$. Using meet irreducibility of $\delta \vee \rho$ and distributivity of the lattice $I[\rho, 1]$, (see (12.4)), we get that $\alpha_{i} \leq \delta \vee \rho$ for some $i=1, \ldots, n$. Without loss of generality we may assume that $\alpha_{1} \leq \delta \vee \rho$. This in particular gives $\alpha_{1}^{2} \leq(\delta \vee \rho)^{2}=\delta^{2} \leq \delta$. Since $\alpha_{1}$ is not the smallest element in the set $\left\{\alpha_{1}, \ldots, \alpha_{n}\right\}$ then, once more without loss of generality, we get $\alpha_{1} \not \leq \alpha_{2}$. This gives $\alpha_{1} \vee \alpha_{2}>\alpha_{2}$ and consequently, by (14.8), $\alpha_{1} \vee \alpha_{2}=\left(\alpha_{1} \vee \alpha_{2}\right)^{2} \vee \bar{\alpha}_{2}=\alpha_{1}^{2} \vee \bar{\alpha}_{2}$. Now, using modularity, distributivity of the subset $\left\{\bar{\alpha}_{2}, \ldots, \bar{\alpha}_{n}\right\}$ and the above equality, we have $\delta \geq \alpha_{1}^{2} \vee\left(\bar{\alpha}_{1} \wedge \ldots \wedge \bar{\alpha}_{n}\right)=$ $\bar{\alpha}_{1} \wedge\left(\alpha_{1}^{2} \vee\left(\bar{\alpha}_{2} \wedge \ldots \wedge \bar{\alpha}_{n}\right)\right)=\bar{\alpha}_{1} \wedge\left(\alpha_{1}^{2} \vee \bar{\alpha}_{2}\right) \wedge \ldots \wedge\left(\alpha_{1}^{2} \vee \bar{\alpha}_{n}\right)=\bar{\alpha}_{1} \wedge\left(\alpha_{1} \vee \alpha_{2}\right) \wedge$ $\left(\alpha_{1}^{2} \vee \bar{\alpha}_{3}\right) \wedge \ldots \wedge\left(\alpha_{1}^{2} \vee \bar{\alpha}_{n}\right)=\bar{\alpha}_{1} \wedge\left(\alpha_{1}^{2} \vee \bar{\alpha}_{3}\right) \wedge \ldots \wedge\left(\alpha_{1}^{2} \vee \bar{\alpha}_{n}\right) \geq \bar{\alpha}_{1} \wedge \bar{\alpha}_{3} \wedge \ldots \wedge \bar{\alpha}_{n}$. Applying the induction hypothesis we are done.

\section{The Representation Theorem}

In this section we prove the following.

Theorem 18. Let $\mathcal{V}$ be a variety satisfying the conditions $(\mathbf{m} . \mathbf{1})-(\mathbf{m} . \mathbf{3 . 5})$. Then there is a finite algebra $\mathbf{D} \in \mathcal{V}$ such that each finite algebra $\mathbf{A} \in \mathcal{V}$ is isomorphic to $\mathbf{D}\left[T_{+}, \Theta, M\right]$ for some dual tree $T_{+}$, a monotonic mapping $\Theta: T_{+} \longrightarrow \operatorname{Con}(\mathbf{D})$, and $a\left(\mathcal{V}, \mathbf{D}, T_{+}, \Theta\right)$-admissible family $M=\left\{M_{\varphi}: \varphi \in T_{+}\right\}$of modules with the property that every quotient of the form $\mathbf{D} / \Theta_{\varphi}$ has no subdirectly irreducible homomorphic image with Abelian monolith and for each $\varphi \in T_{+}$the $\left(\mathbf{D} / \Theta_{\varphi}\right)$-expanded $R\left(\mathcal{V} *\left(\mathbf{D} / \Theta_{\varphi}\right),\left|D / \Theta_{\varphi}\right|\right)-\operatorname{module} M_{\varphi}$ belongs to $\mathcal{V}_{\mathbf{D} / \Theta_{\varphi}}$.

In the proof of the above theorem we will use the following two lemmas. The first lemma is a version of the Chinese Remainder Theorem (see e.g. Ch.5 Ex.68 of [8]), while the second one was shown in [10].

Lemma 19 (Chinese Remainder Theorem). Let $S$ be a distributive (in the sense of Definition 16) subset of a lattice $\operatorname{Con}(\mathbf{A})$ such that the congruences from the meet subsemilattice of $\operatorname{Con}(\mathbf{A})$ generated by $S$ permute. Then for any natural number $n$, all $\psi_{1}, \ldots, \psi_{n} \in S$ and all $a_{1}, \ldots, a_{n} \in A$ such that

$$
\left(a_{i}, a_{j}\right) \in \psi_{i} \vee \psi_{j} \quad \text { for all } i, j=1, \ldots, n
$$

there is an element $a \in A$ satisfying

$$
\left(a, a_{i}\right) \in \psi_{i} \quad \text { for all } i=1, \ldots, n .
$$

Lemma 20. Let $\mathcal{V}$ be a variety with finitely many (up to isomorphism) subdirectly irreducible algebras, all of which are finite. Then there is a finite algebra $\mathbf{S}$ and a class $\mathcal{M}$ of epimorphisms from $\mathbf{S}$ onto subdirectly irreducibles of $\mathcal{V}$, such that:

(i) every subdirectly irreducible algebra in $\mathcal{V}$ is a homomorphic image of $\mathbf{S}$ under some mapping from $\mathcal{M}$,

(ii) if $\sigma: \mathbf{S} \longrightarrow \mathbf{A}$ belongs to $\mathcal{M}$, and $\lambda: \mathbf{A} \longrightarrow \mathbf{B}$ is an isomorphism then $\lambda \circ \sigma \in \mathcal{M}$

(iii) for all subdirectly irreducible algebras $\mathbf{A}, \mathbf{B} \in \mathcal{V}$ and all epimorphisms $\rho$ : $\mathbf{A} \longrightarrow \mathbf{B}, \quad \sigma: \mathbf{S} \longrightarrow \mathbf{B}$ with $\sigma \in \mathcal{M}$ there is an epimorphism $\tau: \mathbf{S} \longrightarrow \mathbf{A}$, such that $\tau \in \mathcal{M}$ and $\rho \circ \tau=\sigma$.

Now we are able to prove Theorem 18. 
Proof. Since the variety $\mathcal{V}$ is finitely generated and residually small, then by Theorem 10.15 of R.Freese and R.McKenzie [7], $\mathcal{V}$ has finitely many (up to isomorphism) subdirectly irreducible algebras and all of them are finite. Thus we can apply Lemma 20 to get a finite algebra $\mathbf{D} \in \mathcal{V}$ and a class $\mathcal{M}$ of epimorphisms for which the conditions (i), (ii) and (iii) of Lemma 20 hold. The epimorphisms from $\mathcal{M}$ will be called admissible ones.

For every finite algebra $\mathbf{A}$ from the variety $\mathcal{V}$ we will construct a monotonic function

$$
\Theta: T_{+} \longrightarrow \operatorname{Con}(\mathbf{D}),
$$

where $T_{+}=\{\varphi \in \operatorname{Cm}(\mathbf{A}): \varphi \geq \rho\} \cup\{1\}, \rho$ is the solvable radical of $\mathbf{A}$, and a family of isomorphisms

such that

$$
\lambda_{\varphi}: \mathbf{A} / \varphi \longrightarrow \mathbf{D} / \Theta_{\varphi}
$$

(0) for every meet irreducible congruence $\varphi \in T_{+}$, the natural epimorphism $\operatorname{nat}_{\varphi}$ is admissible,

(1) if $\varphi \subseteq \psi$ are in $T_{+}$then $\Theta_{\varphi} \subseteq \Theta_{\psi}$ and $\operatorname{nat}\left(\Theta_{\psi} / \Theta_{\varphi}\right) \circ \lambda_{\varphi}=\lambda_{\psi} \circ \operatorname{nat}(\psi / \varphi)$.

Our construction will be done by a downward induction on the position of a congruence $\varphi$ in the dual tree $T_{+}$. If $\varphi=1_{\mathbf{A}}$ then put $\Theta_{\varphi}=1_{\mathbf{D}}$.

Let $\varphi$ be a maximal meet-irreducible congruence in $T_{+}$. By (i) of Lemma 20 there exists an admissible epimorphism $\delta_{\varphi}: \mathbf{D} \longrightarrow \mathbf{A} / \varphi$. Put $\Theta_{\varphi}=k e r \delta_{\varphi}$, and define $\lambda_{\varphi}$ to be the unique homomorphism of the form $\mathbf{A} / \varphi \longrightarrow \mathbf{D} / \Theta_{\varphi}$, for which $\lambda_{\varphi} \circ \delta_{\varphi}=\operatorname{nat}_{\varphi}$. As $\operatorname{ker} \delta_{\varphi}=\operatorname{ker}\left(\right.$ nat $\left.\Theta_{\varphi}\right)$, then $\lambda_{\varphi}$ is an isomorphism, and from (ii) of Lemma 20 we get that $n a t \Theta_{\varphi}$ is admissible.

Now let $\varphi \in T_{+}$be a non-maximal meet irreducible congruence of $\mathbf{A}$ with unique cover $\varphi_{+}$. By the induction hypothesis we have a congruence $\Theta_{\varphi_{+}}$on $\mathbf{D}$ and an isomorphism $\lambda_{\varphi_{+}}: \mathbf{A} / \varphi_{+} \longrightarrow \mathbf{D} / \Theta_{\varphi_{+}}$, such that nat $\Theta_{\varphi_{+}}$is admissible. Since both $\mathbf{A} / \varphi$ and $\mathbf{D} / \Theta_{\varphi_{+}}$are subdirectly irreducible then we can apply (iii) of Lemma 20 to get an admissible epimorphism $\delta_{\varphi}: \mathbf{D} \longrightarrow \mathbf{A} / \varphi$, such that

(2) $\operatorname{nat}\left(\Theta_{\varphi_{+}}\right)=\lambda_{\varphi_{+}} \circ \operatorname{nat}\left(\varphi_{+} / \varphi\right) \circ \delta_{\varphi}$.

Define $\Theta_{\varphi}=\operatorname{ker}_{\varphi}$ and put $\lambda_{\varphi}$ to be the unique isomorphism $\lambda_{\varphi}: \mathbf{A} / \varphi \longrightarrow \mathbf{D} / \Theta_{\varphi}$, for which

(3) $\operatorname{nat}_{\varphi}=\lambda_{\varphi} \circ \delta_{\varphi}$.

It is clear that our construction fulfills the condition (0). To prove that (1) holds, it suffices to show it only in the case when $\psi$ covers $\varphi$, i.e. $\psi=\varphi_{+}$. From (2) we get

$$
\Theta_{\varphi_{+}}=\operatorname{ker}\left(n a t \Theta_{\varphi_{+}}\right) \supseteq \operatorname{ker} \delta_{\varphi}=\Theta_{\varphi} .
$$

Moreover, using (2) and (3) we have

$$
\begin{aligned}
\lambda_{\varphi_{+}} \circ \operatorname{nat}\left(\varphi_{+} / \varphi\right) \circ \delta_{\varphi} & =\operatorname{nat} \Theta_{\varphi_{+}} \\
& =\operatorname{nat}\left(\Theta_{\varphi_{+}} / \Theta_{\varphi}\right) \circ \operatorname{nat} \Theta_{\varphi}=\operatorname{nat}\left(\Theta_{\varphi_{+}} / \Theta_{\varphi}\right) \circ \lambda_{\varphi} \circ \delta_{\varphi}
\end{aligned}
$$

and consequently

$$
\lambda_{\varphi_{+}} \circ \operatorname{nat}\left(\varphi_{+} / \varphi\right)=\operatorname{nat}\left(\Theta_{\varphi_{+}} / \Theta_{\varphi}\right) \circ \lambda_{\varphi}
$$

as $\delta_{\varphi}$ is a surjective map. This proves the correctness of our construction.

Now observe that for each $\varphi \in T_{+}$the congruence $\varphi / \bar{\varphi}$ is the largest Abelian congruence in the quotient $\mathbf{A} / \bar{\varphi}$. Moreover, by $(14.9), I[\bar{\varphi}, 1]=I[\bar{\varphi}, \varphi] \cup I[\varphi, 1]$, and therefore, by Theorem $5, \mathbf{A} / \bar{\varphi}$ is isomorphic to the algebra $(\mathbf{A} / \varphi)\left[M_{\varphi}\right]$ for some $(\mathbf{A} / \varphi)$-expanded $R(\mathcal{V} *(\mathbf{A} / \varphi),|A / \varphi|)$-module $M_{\varphi}$ from $\mathcal{V}_{\mathbf{A} / \varphi}$. Treating $M_{\varphi}$ 
as a $\left(\mathbf{D} / \Theta_{\varphi}\right)$-expanded $R\left(\mathcal{V} *\left(\mathbf{D} / \Theta_{\varphi}\right),\left|D / \Theta_{\varphi}\right|\right)$-module from $\mathcal{V}_{\mathbf{D} / \Theta_{\varphi}}$ we have an isomorphism

$$
\Lambda_{\varphi}: \mathbf{A} / \bar{\varphi} \longrightarrow\left(\mathbf{D} / \Theta_{\varphi}\right)\left[M_{\varphi}\right] \subseteq\left(D / \Theta_{\varphi}\right) \times M_{\varphi}
$$

such that for any $a \in A$

$$
\Lambda_{\varphi}(a / \bar{\varphi})=\left(\lambda_{\varphi}(a / \varphi), \mu_{\varphi}(a / \bar{\varphi})\right),
$$

for some mapping $\mu_{\varphi}: A / \bar{\varphi} \longrightarrow M_{\varphi}$.

On the other hand (14.12) assures us that the natural mapping

$$
\mathbf{A} \longrightarrow \prod_{\varphi \in T_{+}} \mathbf{A} / \bar{\varphi}
$$

is a subdirect embedding, and consequently we get the following subdirect representation

$$
\mathbf{A} \longrightarrow \prod_{\varphi \in T_{+}}\left(\mathbf{D} / \Theta_{\varphi}\right)\left[M_{\varphi}\right]
$$

for the $\left(\mathcal{V}, \mathbf{D}, T_{+}, \Theta\right)$-admissible family $M=\left\{M_{\varphi}: \varphi \in T_{+}\right\}$of modules. An easy calculation shows that this embedding takes $\mathbf{A}$ into $\mathbf{D}\left[T_{+}, \Theta, M\right]$.

To finish the proof that $\mathbf{A}$ is isomorphic to $\mathbf{D}\left[T_{+}, \Theta, M\right]$ we have to show that for each $\alpha \in D^{T_{+}}$and $m \in M=\prod_{\varphi \in T_{+}} M_{\varphi}$ satisfying and

(4) if $\varphi \subseteq \psi$ then $\left(\alpha_{\varphi}, \alpha_{\psi}\right) \in \Theta_{\psi}$

(5) $\left(\alpha_{\varphi} / \Theta_{\varphi}, m_{\varphi}\right) \in\left(D / \Theta_{\varphi}\right)\left[M_{\varphi}\right], \quad$ for all $\varphi \in T_{+}$, there is an element $a$ in the algebra $\mathbf{A}$ with

(6) $\alpha / \Theta_{\varphi}=\lambda_{\varphi}(a / \varphi)$ and $m_{\varphi}=\mu_{\varphi}(a / \bar{\varphi})$ for all $\varphi \in T_{+}$.

Obviously, for each $\varphi \in T_{+}$there is $a_{\varphi} \in A$ such that

$$
\left(\alpha_{\varphi} / \Theta_{\varphi}, m_{\varphi}\right)=\left(\lambda_{\varphi}\left(a_{\varphi} / \varphi\right), \mu_{\varphi}\left(a_{\varphi} / \bar{\varphi}\right)\right) .
$$

Since for all $\varphi, \psi \in T_{+}$we have $\varphi \vee \psi \in T_{+}$, then using (1) we get

$$
\begin{aligned}
\left(\lambda_{\varphi \vee \psi} \circ \operatorname{nat}(\varphi \vee \psi)\right)\left(a_{\varphi}\right) & =\left(\lambda_{\varphi \vee \psi} \circ \operatorname{nat}((\varphi \vee \psi) / \varphi) \circ \operatorname{nat} \varphi\right)\left(a_{\varphi}\right) \\
=\left(\operatorname{nat}\left(\Theta_{\varphi \vee \psi} / \Theta_{\varphi}\right) \circ \lambda_{\varphi} \circ \operatorname{nat} \varphi\right)\left(a_{\varphi}\right) & =\operatorname{nat}\left(\Theta_{\varphi \vee \psi} / \Theta_{\varphi}\right)\left(\lambda_{\varphi}\left(a_{\varphi} / \varphi\right)\right) \\
=\operatorname{nat}\left(\Theta_{\varphi \vee \psi} / \Theta_{\varphi}\right)\left(\alpha_{\varphi} / \Theta_{\varphi}\right) & =\alpha_{\varphi} / \Theta_{\varphi \vee \psi},
\end{aligned}
$$

and analogously

$$
\left(\lambda_{\varphi \vee \psi} \circ \operatorname{nat}(\varphi \vee \psi)\right)\left(a_{\psi}\right)=\alpha_{\psi} / \Theta_{\varphi \vee \psi}
$$

Since, by (4),

$$
\alpha_{\varphi} / \Theta_{\varphi \vee \psi}=\alpha_{\psi} / \Theta_{\varphi \vee \psi} .
$$

and $\lambda_{\varphi \vee \psi}$ is an isomorphism then

$$
\left(a_{\varphi}, a_{\psi}\right) \in \varphi \vee \psi,
$$

which together with (14.10) gives

$$
\left(a_{\varphi}, a_{\psi}\right) \in \bar{\varphi} \vee \bar{\psi}
$$

Now, using the condition (m.1), Theorem 17 and Lemma 19 we get an element $a \in A$ with

$$
\left(a, a_{\varphi}\right) \in \bar{\varphi} \text { for all } \varphi \in T_{+} .
$$

Therefore for all $\varphi \in T_{+}$we have

$$
\alpha_{\varphi} / \Theta_{\varphi}=\lambda_{\varphi}\left(a_{\varphi} / \varphi\right)=\lambda_{\varphi}(a / \varphi)
$$


and

$$
m_{\varphi}=\mu\left(a_{\varphi} / \bar{\varphi}\right)=\mu(a / \bar{\varphi})
$$

as required in (6).

This finishes the proof of Theorem 18.

\section{Proof of the Main Theorem}

First note that, for a finite algebra $\mathbf{D}$ from $\mathcal{V}$ the decidability of the monadic second order theory of finite dual $\mathbf{D}$-trees follows from Lemma 7 and the result of M.O.Rabin [21] stating that the monadic second order theory of the full binary tree with two successor functions is decidable.

Now, we get the sufficiency of the conditions $(\mathbf{m . 1})-(\mathbf{m} .4)$ directly from Corollary 11 and Theorem 18.

The necessity of $(\mathbf{m . 1}),(\mathbf{m . 2}),(\mathbf{m} .3 .1),(\mathbf{m . 3 . 2}),(\mathbf{m} .3 .4)$ and $(\mathbf{m . 3 . 5})$ was obtained by Joohee Jeong in his Ph.D. thesis [14] (see also [15, 16]), while necessity of (m.3.3) was shown by Matthew Valeriote and the present author in [13].

Thus, to finish the proof we need to show that in finitely generated congruence modular varieties the condition (m.4) holds.

Fix a finite algebra $\mathbf{D} \in \mathcal{V}$ having linearly ordered congruences and non-Abelian monolith. We will show the finite decidability of the variety $\mathcal{V}_{\mathbf{D}}$ of $\mathbf{D}$-expanded $R(\mathcal{V} * \mathbf{D},|D|)$-modules by interpreting finite modules from $\mathcal{V}_{\mathbf{D}}$ into finite algebras from $\mathcal{V} * \mathbf{D}$. Since $\mathcal{V} * \mathbf{D}$ is finitely generated, congruence modular and residually small then we can apply a result of R.McKenzie [17], to get that $\mathcal{V} * \mathbf{D}$ is finitely axiomatizable and thus finitely decidable.

In this interpretation we will use another result of R.McKenzie [18], saying that in finitely generated congruence modular varieties each algebra has a largest solvable congruence (which is to be called the solvable radical) and there is a first order formula $\operatorname{Rad}(x, y)$ such that for each algebra $\mathbf{A}$ from the variety and $a, b \in A$

$$
\mathbf{A} \models \operatorname{Rad}(a, b) \quad \text { iff } \quad(a, b) \text { belongs to the solvable radical of } \mathbf{A} .
$$

Let $\left\{d_{1}, \ldots, d_{m}\right\}$ be the universe of $\mathbf{D}$. By Theorem $5, \mathbf{D}[M]$ belongs to $\mathcal{V} * \mathbf{D}$ for each $M \in \mathcal{V}_{\mathbf{D}}$. We can uniformly recover $M$ from $\mathbf{D}[M]^{m}$ by using constants $d_{1}, \ldots, d_{m}$, interpreted in $\mathbf{D}[M]$ by $\left(d_{1}, 0\right), \ldots,\left(d_{m}, 0\right)$ respectively, and the following formulas:

$$
\begin{aligned}
& \Delta\left(x_{1}, \ldots, x_{m}\right) \equiv \boldsymbol{\operatorname { R a d }}\left(x_{1}, d_{1}\right) \& \ldots \& \operatorname{Rad}\left(x_{m}, d_{m}\right), \\
& \operatorname{Plus}(\mathbf{x}, \mathbf{y}, \mathbf{z}) \equiv \bigwedge_{i=1}^{m}\left(z_{i}=\mathbf{p}\left(x_{i}, d_{i}, y_{i}\right)\right) \\
& \operatorname{Times}_{\mathbf{r}}(\mathbf{x}, \mathbf{y}) \equiv \bigwedge_{i=1}^{m}\left(y_{i}=\sum_{j=1}^{m} \mathbf{r}_{i j}\left(x_{i}, d_{1}, \ldots, d_{m}\right)\right)
\end{aligned}
$$

where $\mathbf{x}=\left(x_{1}, \ldots, x_{m}\right), \mathbf{y}=\left(y_{1}, \ldots, y_{m}\right), \mathbf{z}=\left(z_{1}, \ldots, z_{m}\right), \mathbf{p}(x, y, z)$ is a Gumm term for the variety $\mathcal{V}$, the addition in the expression

$$
\sum_{j=1}^{m} \mathbf{r}_{i j}\left(x_{i}, d_{1}, \ldots, d_{m}\right)
$$

is defined by $\mathbf{p}\left(\cdot, d_{i}, \cdot\right)$ and $\mathbf{r}=\left(\mathbf{r}_{i j}\right)$ is an arbitrary matrix from the ring $R(\mathcal{V} * \mathbf{D},|D|)$. 
Now, if $\rho$ is the solvable (= Abelian) radical of $\mathbf{D}[M]$ then Theorem 5 guarantees that $M \simeq \bar{M}_{\mathbf{D}[M]}(\rho)$. The module $M_{\mathbf{D}[M]}(\rho)$ can be easily shown to be isomorphic to the one with the universe determined in $\mathbf{D}[M]^{m}$ by the formula $\Delta$ and operations given by Plus and Times .

Finally we have to recover constants $\mathbf{f}^{(M)}\left(d_{i_{1}}, \ldots, d_{i_{k}}\right)$. Obviously, the element $\mathbf{f}^{(M)}\left(d_{i_{1}}, \ldots, d_{i_{k}}\right)$ corresponds to $\mathbf{f}^{\left.\overline{(}_{\mathbf{D}[M]}(\rho)\right)}\left(d_{i_{1}}, \ldots, d_{i_{k}}\right)$. Using the definition of $\bar{M}_{\mathbf{D}[M]}(\rho)$ given in the proof of Lemma 3 , we know that then $\mathbf{f}^{(M)}\left(d_{i_{1}}, \ldots, d_{i_{k}}\right)$ corresponds to the unique element $\left(u_{1}, \ldots, u_{m}\right)$ of $\mathbf{D}[M]^{m}$ satisfying the formula

$$
\bigvee_{j=1}^{m}\left(\left(\bigwedge_{i \neq j} u_{i}=d_{i}\right) \& u_{j}=\mathbf{f}\left(d_{i_{1}}, \ldots, d_{i_{k}}\right) \& \operatorname{Rad}\left(d_{j}, \mathbf{f}\left(d_{i_{1}}, \ldots, d_{i_{k}}\right)\right)\right) \text {. }
$$

This finishes the proof of the Main theorem.

\section{Concluding Remarks}

In his unpublished manuscript M.Albert [1] reduced the finite decidability of some finitely generated congruence permutable varieties in which all subdirect irreducibles have linearly ordered congruences to the finite decidability of their largest abelian subvarieties. Varieties he was working with satisfy conditions (m.2) and (m.3) since the centralizer of the monolith of each subdirectly irreducible algebra was assumed to be not bigger than the monolith itself. This allows one to think of the abelian part in the congruence lattice of each finite algebra from the variety to be "semisimple" i.e. this part is a direct product of abelian parts - which are simple - of subdirectly irreducibles. This however, is not the case in the general situation.

Moreover, M.Albert supposed that it was unlikely to reduce the finite decidability of locally finite congruence modular varieties to the finite decidability of the largest abelian subvarieties. Here we describe a construction which allows us to confirm his supposition.

Let $\mathbf{A}$ be a finite algebra. We assume that $\mathbf{A}$ has term definable constant 1 . This is not a serious assumption - our construction will work even without it - but we are going to adopt it in order to avoid several technical difficulties. Now choose a new element $0 \notin A$ and extend the universe $A$ of $\mathbf{A}$ to $A \oplus=A \cup\{0\}$. Each $k$-ary operation $\mathbf{f}$ of $\mathbf{A}$ is extended to $A \oplus$ by putting

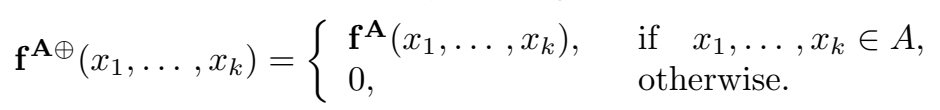

Now $\mathbf{A} \oplus$ denotes this new algebra endowed with an additional binary operation . (written sometimes by a juxtaposition) defined by:

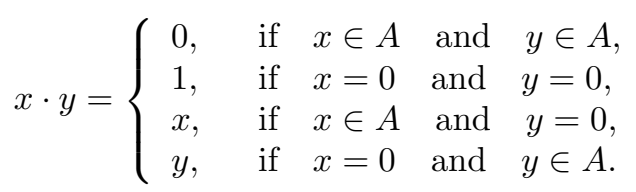

First observe that

(7.1) $\operatorname{Con}(\mathbf{A} \oplus)$ is isomorphic to $\operatorname{Con}(\mathbf{A})$ with a new greatest element adjoined.

Indeed, if $\theta$ is a congruence of $\mathbf{A} \oplus$ then $\theta \cap A^{2}$ is a congruence of $\mathbf{A}$ since $\mathbf{A}$ is a subreduct of $\mathbf{A} \oplus$. Moreover assume that $\theta_{1} \cap A^{2}=\theta_{2} \cap A^{2}$ for two different congruence $\theta_{1}, \theta_{2}$ from $\operatorname{Con}(\mathbf{A} \oplus)-\left\{1_{\mathbf{A} \oplus}\right\}$. Without loss of generality we can 
assume that there is $a \in A$ with $(0, a) \in \theta_{1}-\theta_{2}$. Then for each $b \in A$ we have $b=b \cdot 0 \stackrel{\theta_{1}}{=} a \cdot b=0$ and therefore $\theta_{1}=1_{\mathbf{A} \oplus}$. This contradiction shows that the mapping

$$
\operatorname{Con}(\mathbf{A} \oplus)-\left\{1_{\mathbf{A} \oplus}\right\} \ni \theta \longmapsto \theta \cap A^{2} \in \operatorname{Con}(\mathbf{A})
$$

is a lattice embedding. Moreover it is easy to see that for $\varphi \in \operatorname{Con}(\mathbf{A}), \varphi \cup\{(0,0)\} \in$ $\operatorname{Con}(\mathbf{A} \oplus)$ which shows (7.1).

Let $1_{\mathbf{A}}$ denote the unique coatom in $\operatorname{Con}(\mathbf{A} \oplus)$. The next observation is formulated in the language of Tame Congruence Theory (see [9]).

(7.2) The prime quotient $\left(1_{\mathbf{A}}, 1\right)$ is of the type $\mathbf{3}$ and for any $\alpha \prec \beta<1$ from $\operatorname{Con}(\mathbf{A} \oplus), \operatorname{typ}(\alpha, \beta)=\operatorname{typ}\left(\alpha \cap A^{2}, \beta \cap A^{2}\right)$.

The second claim is obvious since the new operation - is trivial on $A$, while the first one follows easily from the fact that $p(x)=(x x)(x x)$ is an idempotent polynomial on $\mathbf{A} \oplus$ with range $\{0,1\} \in M_{\mathbf{A} \oplus}\left(1_{\mathbf{A}}, 1\right)$, and the operation $\cdot$ acts on $\{0,1\}$ as the Sheffer function.

Now in the algebra $\mathbf{A} \oplus$ we introduce the following terms:

$$
\begin{aligned}
x \wedge y & =(x y)(x y), \\
\neg x & =x x, \\
x \vee y & =\neg(\neg x \wedge \neg y), \\
x+y & =((x y) y)((y x) x), \\
0 & =x+x, \\
1 & =00, \\
x * y & =((((((x y) 1) 1) x) 1)+y)+x .
\end{aligned}
$$

For a subalgebra $\mathbf{D}$ of the power $(\mathbf{A} \oplus)^{X}$ by $D(0,1)$ we denote the set $\{a \in D$ : $\left.a^{-1}(X) \subseteq\{0,1\}\right\}$. An easy calculation shows that:

(7.3) If $\mathbf{D} \leq(\mathbf{A} \oplus)^{X}, a \in D(0,1)$ and $x \in D$ then $x=(a * x)+(\neg a * x)$.

Given $Z \subseteq X$ by $\eta_{Z}$ we denote the kernel of the projection $\mathbf{D} \longrightarrow(\mathbf{A} \oplus)^{Z}$, i.e. $(a, b) \in \eta_{Z}$ iff $a, b \in D$ and $Z \subseteq \llbracket a=b \rrbracket$. Then we have:

(7.4) If $\mathbf{D} \leq(\mathbf{A} \oplus)^{X}$ and $a \in D(0,1)$ then:

(7.4.1) $\Theta_{\mathbf{D}}(a, 0)=\eta_{\llbracket a=0 \rrbracket}$,

(7.4.2) $\left(\Theta_{\mathbf{D}}(a, 0), \Theta_{\mathbf{D}}(\neg a, 0)\right)$ is a factor pair of congruences,

(7.4.3) $D / \Theta_{\mathbf{D}}(a, 0) \leq(\mathbf{A} \oplus)^{\llbracket a=0 \rrbracket}$.

Obviously $(a, 0) \in \eta_{\llbracket a=0 \rrbracket}$ and thus $\Theta_{\mathbf{D}}(a, 0) \subseteq \eta_{\llbracket a=0 \rrbracket}$. To see the converse inclusion, fix $(c, d) \in \eta_{\llbracket a=0 \rrbracket}$ and observe that then $a * c=a * d$. Moreover note that for all $x \in D$ we have $(0, \neg a * x)=(1 * x, \neg a * x) \in \Theta_{\mathbf{D}}(a, 0)$. In particular $(\neg a * c, \neg a * d) \in \Theta_{\mathbf{D}}(a, 0)$. Now using (7.3) we get

$$
(c, d)=((a * c)+(\neg a * c),(a * d)+(\neg a * d)) \in \Theta_{\mathbf{D}}(a, 0) .
$$

Thus (7.4.1) is shown.

Now $\Theta_{\mathbf{D}}(a, 0) \cap \Theta_{\mathbf{D}}(\neg a, 0)=0$, and for (7.4.2) it remains to show that $\Theta_{\mathbf{D}}(a, 0) \circ$ $\Theta_{\mathbf{D}}(\neg a, 0)=1$. To see this fix $c, d \in D$ and put $e=(a * c)+(\neg a * d)$. Obviously $e \in D$ and, by (7.4.1), we obtain $(c, e) \in \Theta_{\mathbf{D}}(a, 0)$ and $(e, d) \in \Theta_{\mathbf{D}}(\neg a, 0)$, as required.

To see (7.4.3), it suffices to note that $\Theta_{\mathbf{D}}(a, 0)$ is the kernel of the projection

$$
\left.\mathbf{D} \ni x \longmapsto x\right|_{\llbracket a=0 \rrbracket} \in(\mathbf{A} \oplus)^{\llbracket a=0 \rrbracket} .
$$


Now, if $\mathbf{D} \leq(\mathbf{A} \oplus)^{X}$ is such that $D(0,1)=\{0,1\}$ then it can be easily seen that $D-\{0\}$ is a subuniverse of $\mathbf{A}^{X}$. Moreover for $x, y \in D$ we have

$$
x \cdot y=\left\{\begin{array}{lll}
0, & \text { if } x \neq 0 \text { and } y \neq 0 \\
1, & \text { if } x=0 \text { and } y=0 \\
x, & \text { if } x \neq 0 \text { and } y=0 \\
y, & \text { if } x=0 \text { and } y \neq 0
\end{array}\right.
$$

Consequently $\mathbf{D}$ is isomorphic to $\mathbf{B} \oplus$ for some $\mathbf{B} \in S P_{\text {fin }}(\mathbf{A})$. Combining this with (7.1) and (7.4) we immediately get

(7.5) If $\mathbf{D} \in S P_{\text {fin }}(\mathbf{A} \oplus)$ then $\mathbf{D}$ is directly indecomposable iff $D(0,1)=\{0,1\}$. Moreover every algebra from $S P_{f i n}(\mathbf{A} \oplus)$ is a direct product of directly indecomposables from $S P_{\text {fin }}(\mathbf{A} \oplus)$.

Now, let $\mathcal{A}=\left\{\mathbf{B} \oplus: \mathbf{B} \in H S P_{\text {fin }}(\mathbf{A})\right\}$. We already know that $\mathcal{A}$ contains all directly indecomposable algebras from $S P_{\text {fin }}(\mathbf{A} \oplus)$. Actually we will show that $\mathcal{A}$ is the class of all directly indecomposables from $H S P_{f i n}(\mathbf{A} \oplus)$ by proving that

Fact 21. $H S P_{\text {fin }}(\mathbf{A} \oplus)=P_{\text {fin }}(\mathcal{A})$.

Proof. Obviously $\mathbf{B} \in H S P_{\text {fin }}(\mathbf{A})$ implies $\mathbf{B} \oplus \in H S P_{\text {fin }}(\mathbf{A} \oplus)$, and thus $P_{\text {fin }}(\mathcal{A})$ $\subseteq H S P_{\text {fin }}(\mathbf{A} \oplus)$. Conversely, by the remarks below (7.5) we already know that $S P_{\text {fin }}(\mathbf{A} \oplus) \subseteq P_{f i n}(\mathcal{A})$. To see that each quotient $\mathbf{D} / \theta$ of an algebra from $S P_{f i n}(\mathbf{A} \oplus)$ belongs to $P_{f i n}(\mathcal{A})$ it suffices to show this with an additional assumption saying that $\theta$ is an atom in $\operatorname{Con}(\mathbf{D})$.

First we show that if $\mathbf{D}$ is treated as a finite direct product $\prod_{t \in T} \mathbf{A}_{t}$ of algebras from $\mathcal{A}$ then the atomic congruence $\theta$ is contained in some projection kernel of the form $\eta_{T-\{t\}}$. Assume to the contrary that this is not the case. Then we can find $(a, b),(c, d) \in \theta$ and $t, s \in T$ with $t \neq s, a_{t} \neq b_{t}$ and $c_{s} \neq d_{s}$. Since $\mathbf{D}$ is the full direct product of $\mathbf{A}_{t}$ 's then the elements $e^{t}=\left.\left.0\right|_{\{t\}} \cup 1\right|_{T-\{t\}}$ and $e^{s}=\left.\left.0\right|_{\{s\}} \cup 1\right|_{T-\{s\}}$ belong to $\mathbf{D}$. Moreover $\left(e^{t} * a, e^{t} * b\right) \in \theta-0$ and $\left(e^{s} * c, e^{s} * d\right) \in \theta-0$. On the other hand for $\theta^{t}=\Theta_{\mathbf{D}}\left(e^{t} * a, e^{t} * b\right)$ and $\theta^{s}=\Theta_{\mathbf{D}}\left(e^{s} * c, e^{s} * d\right)$ we have $\theta^{t} \subseteq \eta_{T-\{t\}}$ and $\theta^{s} \subseteq \eta_{T-\{s\}}$, and thus $\theta^{t} \cap \theta^{s}=0$. Since $\theta^{t}, \theta^{s}$ are nontrivial congruences below $\theta$ then $\theta^{t}=\theta=\theta^{s}$ and consequently $\theta=\theta^{t} \cap \theta^{s}=0$, a contradiction.

This shows that $\theta$ is contained in some projection kernel of the form $\eta_{T-\{t\}}$. However a quotient of a direct product by the congruence contained in such a projection kernel is isomorphic to a direct product of all $\mathbf{A}_{s}$ 's, $s \neq t$ and a quotient of $\mathbf{A}_{t}$. Now we can finish the proof by noting that (7.1) implies that $\mathcal{A}$ is closed under taking homomorphic images.

Now we are in a position to prove the following:

Theorem 22. If $\mathbf{A}$ is a finite algebra (with term definable constant) then the variety generated by $\mathbf{A}$ is finitely decidable if and only if the variety generated by $\mathbf{A} \oplus$ is finitely decidable.

Proof. The reader will find no difficulties in observing that the decidability of $H S P_{\text {fin }}(\mathbf{A})$ implies the decidability of $\mathcal{A}$. Using a result of A.Mostowski [20] we get that $P_{\text {fin }}(\mathcal{A})$ is decidable which, in view of Fact 21, gives that $\mathbf{A} \oplus$ generates a finitely decidable variety. 
Conversely, if $H S P_{\text {fin }}(\mathbf{A} \oplus)=P_{\text {fin }}(\mathcal{A})$ is decidable then $\mathcal{A}$ is decidable since $\mathcal{A}$ is finitely axiomatizable relative to $P_{f i n}(\mathcal{A})$ by the sentence

$$
\forall x(x=(x x)(x x) \Rightarrow(x=0 \quad \text { or } \quad x=1)) .
$$

Now $H S P_{\text {fin }}(\mathbf{A})$ is trivially interpretable into $\mathcal{A}$, which gives the finite decidability of the variety generated by $\mathbf{A}$.

Theorem 23. There is a finitely generated congruence modular variety satisfying the conditions (m.1)-(m.3) that is finitely undecidable and such that its largest abelian subvariety is finitely decidable.

Proof. First we will show that starting with a finite algebra $\mathbf{A}$ generating a variety $\mathcal{V}$ that satisfies the conditions $(\mathbf{m . 1})-(\mathbf{m} . \mathbf{3})$ the derived variety $\mathcal{V} \oplus=H S P(\mathbf{A} \oplus)$ satisfies these conditions as well.

Indeed, one can check that the term

$p \oplus(x, y, z)=((\neg x \vee \neg y \vee \neg z) * p(x, y, z))+((x \vee y \vee \neg z) * z)+((\neg x \vee y \vee z) * x)$

is a Malcev term for $\mathbf{A} \oplus$ whenever $p(x, y, z)$ is one for $\mathbf{A}$.

Now, observe that the isomorphism between $\operatorname{Con}(\mathbf{B} \oplus)-\left\{1_{\mathbf{B} \oplus}\right\}$ and $\operatorname{Con}(\mathbf{B})$ described in the proof of (7.1) preserves the commutator operation, that is for $\alpha, \beta \in \operatorname{Con}(\mathbf{B} \oplus)$ we have

$$
[\alpha, \beta]_{\mathbf{B} \oplus}= \begin{cases}{\left[\alpha \cap B^{2}, \beta \cap B^{2}\right]_{\mathbf{B}} \cup\{(0,0)\},} & \text { if } \alpha \neq 1_{\mathbf{B} \oplus \neq \beta,} \neq \beta, \\ \beta, & \text { if } \alpha=1_{\mathbf{B} \oplus}, \\ \alpha, & \text { if } \beta=1_{\mathbf{B} \oplus .}\end{cases}
$$

To prove that $\mathcal{V} \oplus$ is residually small it suffices (cf. Thm. 10.15 of [7]) to check that the congruences of any subalgebra of $\mathbf{A} \oplus$ satisfy the identity

$$
\alpha \wedge[\beta, \beta]=[\alpha \wedge \beta, \beta] .
$$

However, if $\mathbf{D}$ is a subalgebra of $\mathbf{A} \oplus$ then, since 0 is a term definable constant, $0 \in D$ and moreover $D-\{0\}$ is a subuniverse of $\mathbf{A}$. Consequently $\mathbf{D}$ is isomorphic to $\mathbf{B} \oplus$ for some subalgebra $\mathbf{B} \subseteq \mathbf{A}$. Using (7.6) and the fact that $\mathbf{B}$ satisfies (7.7) it is easy to see that (7.7) holds in $\operatorname{Con}(\mathbf{B} \oplus)$.

Finally, to check that (m.3) holds in $\mathcal{V} \oplus$ fix a subdirectly irreducible algebra $\mathbf{D} \in \mathcal{V} \oplus$. Then $\mathbf{D}$ is finite (by $(\mathbf{m} . \mathbf{2})$ already established for $\mathcal{V} \oplus$ ) and directly indecomposable, and thus $\mathbf{D}=\mathbf{B} \oplus$ for some $\mathbf{B} \in H S P_{\text {fin }}(\mathbf{A})$ such that $\mathbf{B}$ is either trivial or subdirectly irreducible. If $\mathbf{B}$ is trivial then $\mathbf{D}$ is term equivalent to the two element Boolean algebra and therefore satisfies $(\mathbf{m . 3 . 1})-(\mathbf{m . 3 . 5})$. So, let $\mathbf{B}$ be a subdirectly irreducible algebra from $\operatorname{HSP}(\mathbf{A})$ and let $\nu$ be the centralizer of its monolith. Again, using (7.6), we get that $\nu \oplus=\nu \cup\{(0,0)\}$ is the centralizer of the monolith of $\mathbf{D}$. The facts that $\operatorname{Con}(\mathbf{D})$ is isomorphic to $\operatorname{Con}(\mathbf{B})$ with a new largest element adjoined and that the commutator on $\operatorname{Con}(\mathbf{D})$ is given by (7.6) imply that $\nu \oplus$ satisfies (m.3.1) - (m.3.5).

After this preparation we return to the proof of our theorem. Let $\mathbf{A}$ be a finite module generating a finitely undecidable variety $\mathcal{V}$, (see e.g. [28] for such an example). Then, by Theorem 22, the variety $\mathcal{V} \oplus$ generated by $\mathbf{A} \oplus$ is finitely undecidable. Since each directly indecomposable and thus each subdirectly irreducible algebra from $\mathcal{V} \oplus$ is of the form $\mathbf{B} \oplus$ for some $\mathbf{B} \in \mathcal{V}_{\text {fin }}$ then, by (7.2), it is non-Abelian. This means that the largest Abelian subvariety of $\mathcal{V} \oplus$ is trivial and therefore finitely decidable. 
On the other hand, the variety $\mathcal{V}$, as a finitely generated variety of modules, is residually small, Abelian and congruence permutable. Therefore $\mathcal{V}$, and consequently $\mathcal{V} \oplus$, satisfies $(\mathbf{m . 1})-(\mathbf{m} .3)$.

\section{ACKNOWLEDGMENT}

The author would like to thank Matthew Valeriote and an anonymous referee for very carefully reading the manuscript and pointing out several gaps in the previous versions of it.

\section{REFERENCES}

1. M.H.Albert, A sufficient condition for finite decidability, manuscript, 1991.

2. S.Burris and R.McKenzie, Decidability and Boolean Representation, Memoirs Amer. Math. Soc., 246(1981). MR 83j:03024

3. S.Burris, R.McKenzie and M.Valeriote, Decidable discriminator varieties from unary varieties, Journal of Symbolic Logic, 56(1991), 1355-1368. MR 93f:08006

4. S.Burris and H.P.Sankappanavar, A Course in Universal Algebra, Springer Verlag 1981. MR 83k:08001

5. S.D.Comer, Elementary properties of structures of sections, Bol. Soc. Mat. Mexicana, 19(1974), 78-85. MR 55:10265

6. A.Ehrenfeucht, Decidability of the theory of one function, Notices Amer. Math. Soc., 6(1959), 268.

7. R.Freese and R.McKenzie, Commutator theory for congruence modular varieties, London Math. Soc. Lecture Notes, 125, 1987. MR 89c:08006

8. G.Grätzer, Universal Algebra, 2nd ed., Springer-Verlag, New York 1979. MR 80g:08001

9. D.Hobby and R.McKenzie, The Structure of Finite Algebras, Amer. Math. Soc. Contemporary Mathematics, vol. 76, Providence Rhode Island 1988. MR 89m:08001

10. P.M.Idziak, Reduced sub-powers and the decision problem for finite algebras in arithmetical varieties, Algebra Universalis, 25(1988), 365-383. MR 89k:08005

11. P.M.Idziak, Varieties with decidable finite algebras I: linearity, Algebra Universalis, 26(1989), 234-246. MR 90d:08006a

12. P.M.Idziak, Varieties with decidable finite algebras II: permutability, Algebra Universalis, 26(1989), 247-256. MR 90d:08006b

13. P.M.Idziak and M.Valeriote, A property of the solvable radical in finitely decidable varieties, manuscript, 1991.

14. J.Jeong, On finitely decidable varieties, Ph.D. Thesis, University of California (Berkeley), 1991.

15. J.Jeong, Finitary decidability implies permutability for congruence modular varieties, Algebra Universalis, 29(1992), 441-448. MR 93h:08010

16. J.Jeong, Finitely decidable congruence modular varieties, Trans. Amer. Math. Soc., 339(1993), 623-642. MR 93m:08011

17. R.McKenzie, Finite equational bases for congruence modular varieties, Algebra Universalis, 24(1987), 224-250. MR 89j:08007

18. R.McKenzie, Nilpotent and solvable radicals in locally finite congruence modular varieties, Algebra Universalis, 24(1987), 251-266. MR 89j:08008

19. R.McKenzie and M.Valeriote, The Structure of Decidable Locally Finite Varieties, Birkhäuser, Boston, 1989. MR 92j:08001

20. A.Mostowski, On direct product of theories, Journal of Symbolic Logic, 17(1952), 1-31. MR 13:897a

21. M.O.Rabin, Decidability of second order theories and automata on infinite trees, Trans. Amer. Math. Soc., 141(1969), 1-35. MR 40:30

22. M.O.Rabin, Decidable theories, in: J.D.Barwise ed., Handbook of Mathematical Logic, North Holland 1977, pp. 595-629. MR 56:15351

23. W.Szmielew, Elementary properties of Abelian groups, Fund. Math., 55(1955), 203-271. MR $17: 233 \mathrm{e}$

24. A.Tarski, Arithmetical classes and types of Boolean algebras, Bull. Amer. Math. Soc., $\mathbf{5 5}(1949), 64$. 
25. M.Valeriote and R.Willard, Discriminating varieties, Algebra Universalis, 32(1994), 177-188. MR 95m:08010

26. H.Werner, Discriminator Algebras, Studien zur Algebra und ihre Anwendungen, Band 6, Akademie-Verlag, Berlin 1978. MR 80f:08009

27. R.Willard, Decidable discriminator varieties from unary classes, Trans. Amer. Math. Soc., 336(1993), 311-333. MR 93e:08005

28. R.Willard, Hereditary undecidability of some theories of finite structures, Journal of Symbolic Logic, 59(1994), 1254-1262. MR 95m:03092

29. A.P.Zamyatin, A non-Abelian variety of groups has an undecidable elementary theory, Algebra and Logic, 17(1978), 13-17. MR 80c:03045

30. A.P.Zamyatin, Prevarieties of associative rings whose elementary theory is decidable, Soviet Math. Dokl., 19(1978), 890-901. MR 80i:16049

Computer Science Department, Jagiellonian University, Kraków, Poland

E-mail address: idziak@ii.uj.edu.pl 